\title{
Proyecciones
}

Vol. $12 \mathrm{~N}^{\circ}$ 2, pp. 63-118 Diciembre 1993

Universidad Católica del Norte

Antofagasta - Chile

\section{UN SURVOL SUR LA THÉORIE DE HODGE-DE RHAM DES VARIÉTÉS LISSES ET SINGULIĖRES}

\author{
AZIZ EL KACIMI ALAOUI \\ URA au CNRS 751 GAT Lille I \\ Université de Valenciennes, France
}

\begin{abstract}
L'objet de ce papier est de faire un léger survol sur la cohomologie des formes différentielles: de de Rham et sa représentation par les formes harmoniques dans le cas compact lisse, des formes $L^{2}$ dans le cas singulier et enfin les formes basiques et leur théorie de Hodge pour le cas d'un feuilletage riemannien sur une variété compacte.
\end{abstract}

La version finale de ce travail a été élaborée lors d'un séjour que j’ai effectué à la Universidad Católia del Norte à Antofagasta (Chili) en Juillet et Août 1993. Je voudrais remercier R. Correa, R. Soto et tous les autres membres du Departamento de Matemáticas de la UCN pour leur invitation et l'accueil chaleureux qu'ils m'ont réservé. 


\section{CONTENU}

\section{INTRODUCTION}

65

\section{PREMIÉRE PARTIE}

1. LE POINT DE VUE DE CĔCH 66

2. LE POINT DE VUE SINGULIER 69

3. LA COHOMOlogie DE DE Rifam 71

4. La Dualité De DE RHam $\quad 75$

DEUXIEME PARTIE

5. La COHOMOlogie $L^{2} \quad 78$

6. EXEMPLES DE CALCUL $L^{2} \quad 80$

7. ThÉORIE DE Hodge 85

TROISIÈME PARTIE

8. Feuilletages : DÉfinitions 88

9. Feullletages : EXemples 90

10. FEUilletages ET SYSTÈmes DiffÉRENTIELS 95

11. Structures transverses 97

\section{QUATRIÈME PARTIE}

12. Сohomologie Basique 101

13. Théorie de Hodge Basique 111

RÉFÉRENCES $\quad 116$ 


\section{INTRODUCTION}

La plupart des branches des Mathématiques se donnent comme but principal la classification des objets dont elles usent. Un exemple bien connu (et non trivial) est celui de la classification des surfaces fermées orientables : le genre (i.e le nombre de trous) suffit largement à déterminer topologiquement (et différentiablement) la surface. Mais déjà en dimension 3 le problème reste grandement ouvert et il l'est encore plus en dimension supérieure. Une manière de l'aborder consiste à rechercher des invariants dans une catégorie où le calcul est possible; par exemple la catégorie algébrique (groupes, anneaux, espaces vectoriels etc...). Ce sont les méthodes de la Topologie algébrique qui permettent de faire cela. L'un des invariants les plus connus consiste à associer à un espace topologique $M$ une suite d'espaces vectoriels $\left(H^{r}(M, \mathbb{R})\right)_{r \geq 0}$ appelée la cohomologie réelle de $M$. Cette suite peut être calculée en usant d'objets particuliers sur $M$. Pour la plupart des espaces il existe fondamentalement deux procédés de calcul : un, combinatoire, consistant à recouvrir $M$ par des ouverts dont les intersections finies ont des cohomologies triviales pour $r \geq 1$ (cette condition permet d'écarter toute obstruction au calcul global); c'est le point de vue de Céch. Un autre, dit singulier, passe par une triangulation de $M$.

Mais si $M$ est une variété différentiable on peut utiliser l'espace tangent en chaque point pour introduire un "autre invariant" ( à l'aide des formes différentielles) appelé la cohomologie de de Rham de $M$ et noté $H_{D R}^{*}(M)$. Il se calcule en usant fortement de la structure différentiable mais en réalité il ne dépend que de la topologie de $M$ (en fait du type d'homotopie). Plus même : $H_{D R}^{*}(M)$ est le dual de l'homologie singulière, donc isomorphe à $H^{*}(M, \mathbb{R})$. C'est le Théorème de de Rham; certainement le résultat le plus marquant de la théorie.

Si on suppose $M$ compacte, alors l'usage d'une métrique riemannienne permet de calculer $H_{D R}^{*}(M)$ par des formes différentielles plus jolies que les autres appelées formes har- 
moniques. D'abord elles sont "peu nombreuses" (elles forment un espace vectoriel de dimension finie) ensuite elles "sont déjà" la cohomologie de de Rham (c'est le Théorème de Hodge) : toute classe de cohomologie contient une forme harmonique et une seule.

Il y a des espaces topologiques intéressants ne possédant pas de structure différentiable pour lesquels on a pourtant envie d'établir des théorèmes du même type. Beaucoup d'entre-eux apparaissent comme espaces d'orbites d'actions de groupes ou comme espaces des feuilles sur de vraies variétés différentiables. La connaissance de leurs invariants (de nature cohomologique par exemple) peut donner des renseignements sur ces actions.

Le but de ce texte est de faire un léger survol sur la théorie de Hodge-de Rham dans le cas lisse et le cas singulier. Dans la première partie on donne les ingrédients essentiels qui permettent d'énoncer et de comprendre (l'énoncé) du Théorème de de Rham. On y présente aussi de façon brève et élémentaire la Théorie de Hodge dans le cas compact lisse. Dans la deuxième partie on présente la cohomologie et la Théorie de Hodge $L^{2}$ d'une variété riemannienne complète ainsi que quelques calculs explicites de formes harmoniques. Dans la troisième partie on donne la définition de la notion de feuilletage, de structure transverse avec beaucoup d'exemples afin de pouvoir introduire la Théorie de Hodge basique pour un feuilletage riemannien dans la quatrième partie.

\section{PREMIÈRE PARTIE}

\section{LE POINT DE VUE DE CĚCH}

Soient $M$ un espace topologique et $\mathcal{U}=\left(U_{i}\right)_{i \in I}$ un recouvrement ouvert de $M$.

1.1. Définition. On dira que $\mathcal{U}$ est un bon recouvrement si pour toute partie finie $\left(i_{0}, \ldots, i_{r}\right) \subset I$, l'intersection $U_{i_{o} \ldots i_{r}}=$ $U_{i_{0}} \cap \ldots \cap U_{i_{r}}$ est contractile. 
On dira que $M$ est un bon espace si $M$ possède un bon recouvrement dénombrable. C'est le cas si $M$ est une variété différentiable paracompacte.

Dans toute la suite on ne considérera que les bons espaces. Soit $M$ un tel espace avec un bon recouvrement $\mathcal{U}=\left(U_{i}\right)_{i \in \mathbb{N}}$.

Pour chaque $r \in \mathbb{N}$ on pose

$$
\Sigma_{r}=\left\{\left(i_{0}, \ldots, i_{r}\right) \subset I: U_{i_{0} \ldots i_{r}} \neq \emptyset\right\}
$$

et on note $C^{r}$ l'ensemble de toutes les fonctions réelles sur $\Sigma_{r}$. C'est l'espace vectoriel des cochaînes réelles de degré $r$. On considère l'application linéaire

$$
\delta: C^{r} \longrightarrow C^{r+1}
$$

définie comme suit : si $f \in C^{r}$ alors $\delta f$ sera l'élément de $C^{r+1}$ tel que

$$
\delta f\left(i_{0}, \ldots, i_{r+1}\right)=\sum_{k=0}^{r+1}(-1)^{k+1} f\left(i_{0}, \ldots, \widehat{i}_{k}, \ldots, i_{r+1}\right) .
$$

On vérifie facilement que $\delta^{2}: C^{r-1} \longrightarrow C^{r+1}$ est nulle. Autrement dit l'image $B^{r}$ de $\delta: C^{r-1} \longrightarrow C^{r}$ est un sousespace vectoriel du noyau $Z^{r}$ de $\delta: C^{r} \longrightarrow C^{r+1}$. Le quotient

$$
H^{r}(M, \mathbb{R})=Z^{r} / B^{r}
$$

ne dépend pas $\mathrm{du}$ bon recouvrement choisi $\mathcal{U}$ (théorème de Leray). On l'appelle le $r^{\text {ème }}$ espace vectoriel de cohomologie de Céch de $M$.

1.2. Exemple de calcul. Le cercle $\mathbb{S}^{1}$ peut être vu comme l'image de l'application

$$
\sigma: \theta \in \mathbb{R} \longrightarrow e^{i \theta} \in \mathbb{C} .
$$


Soient $U_{0}, U_{1}$ et $U_{2}$ les ouverts donnés par (voir Fig. 1) : $U_{0}=\sigma(]-\frac{\pi}{3}, \frac{2 \pi}{3}[), U_{1}=\sigma(] \frac{\pi}{3}, \frac{4 \pi}{3}[)$ et $U_{2}=\sigma(] \pi, 2 \pi[)$

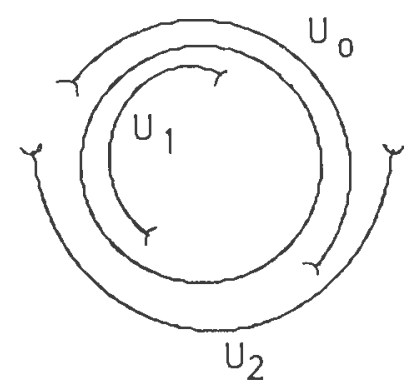

Fig. 1

Il est clair que $=\left(U_{0}, U_{1}, U_{2}\right)$ est un bon recouvrement de $\mathbb{S}^{1}$. On a

$$
\Sigma_{0}=\{0,1,2\}
$$

et donc $C^{0}=\mathbb{R}^{3}$; ensuite

$$
\Sigma_{1}=\{(0,1),(1,2),(0,2)\} .
$$

D'où $C^{1}=\mathbb{R}^{3}$. Comme $\Sigma_{r}=\emptyset$ pour $r \geq 2, C^{r}=0$. Les espaces de cohomologie de Cèch non nuls ne seraient donc éventuellement qu'en degrés 0 et 1 . Nous allons les calculer de manière précise.

Tout élément $f \in C^{0}$ est déterminé par 3 réels $f(0)=f_{0}$, $f(1)=f_{1}$ et $f(2)=f_{2}$. On a $\delta f(i, j)=f_{j}-f_{i}$. Par conséquent $\delta: C^{0} \longrightarrow C^{1}$ est l'application linéaire $\delta: \mathbb{R}^{3} \longrightarrow \mathbb{R}^{3}$ définie par

$$
\delta\left(f_{0}, f_{1}, f_{2}\right)=\left(f_{1}-f_{0}, f_{2}-f_{1}, f_{2}-f_{0}\right) .
$$

Son noyau est donc le sous-espace engendré par le vecteur 
$(1,1,1)$ et son image est un sous-espace de dimension 2 de $\mathbb{R}^{3}$. D'autre part $\delta: C^{1} \longrightarrow C^{2}$ est nulle puisque $C^{2}=0$. D'où

$$
H^{r}\left(\mathbb{S}^{1}, \mathbb{R}\right)= \begin{cases}\mathbb{R} & \text { si } r=0,1 \\ 0 & \text { si } r \geq 2\end{cases}
$$

Désormais pour simplifier on supposera que $M$ est une variété $C^{\infty}$ paracompacte (une classe de différentiabilité plus basse suffira et comme on peut le voir au début du paragraphe qui vient, la différentiabilité n'est même pas nécessaire).

\section{LE POINT DE VUE SINGULIER}

Rappelons un peu comment est faite la cohomologie singulière.

2.1. Les chaînes singulières. On appelle simplexe standard de dimension $r$ l'enveloppe conrexe des points $\left\{v_{0}, \ldots, v_{r}\right\}$ dans l'espace euclidien $\mathbb{R}^{r+1}$ où $v_{k}$ est le $(k+1)^{\text {ème }}$ vecteur de la base canonique. On le notera $\Delta_{r}$ (voir Fig. 2).
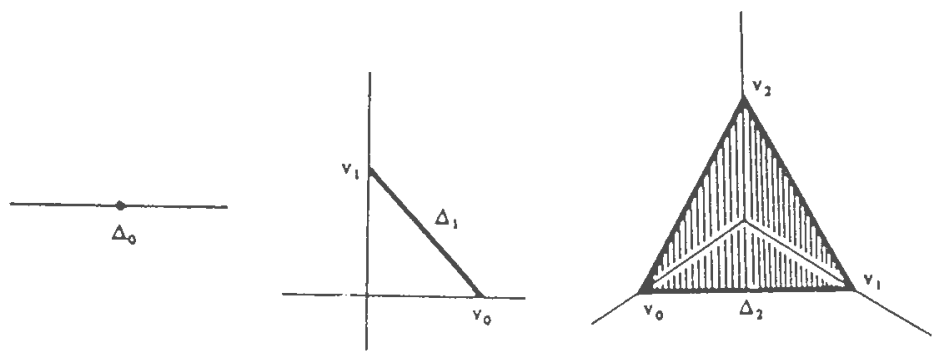

Fig. 2

Une application continue $\phi: \Delta_{r} \longrightarrow M$ est appelée un $r$ simplexe singulier de $M$. Une $r$-chaîne singulière (réelle) est une combinaison linéaire finie à coefficients réels de $r$-simplexes singuliers $\left(\phi_{i}\right)$ :

$$
c=\sum_{i} a_{i} \phi_{i}
$$


L'ensemble $C_{r}^{S}$ des $r$-chaînes singulières est un espace vectoriel réel.

2.2. L'opérateur bord. Soient $\phi$ un $r$-simplexe et $i \in$ $\{0, \ldots, r\}$. On définit le $(r-1)$-simplexe $\partial_{i} \phi$ comme suit (voir Fig. 3) :

$$
\partial_{i} \phi\left(x_{0}, \ldots, x_{r-1}\right)=\phi\left(x_{0}, \ldots, x_{i-1}, 0, x_{i+1}, \ldots, x_{r-1}\right)
$$

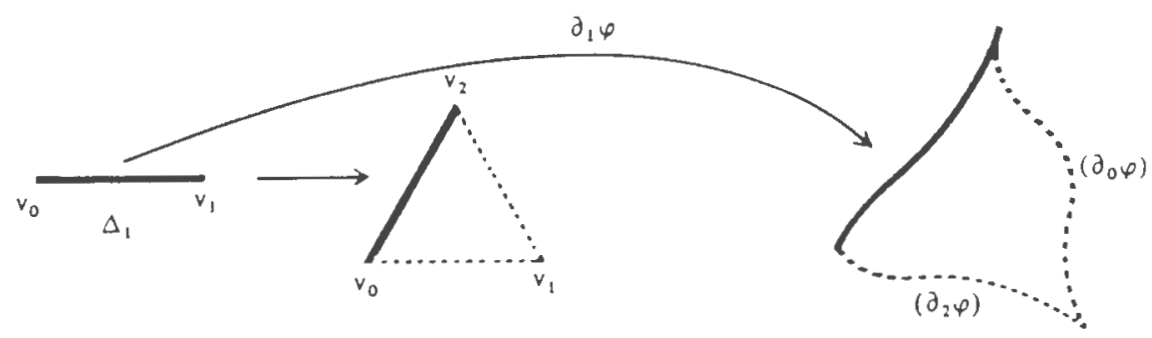

Fig. 3

On pose $\partial=\sum_{i=0}^{r}(-1)^{i} \partial_{i}$. Cet opérateur s'étend (par linéarité) en un opérateur $\partial: C_{r}^{S} \longrightarrow C_{r-1}^{S}$. Un calcul facile à mener montre que $\partial$ est de carré nul.

Désignons par $C_{S}^{r}$ le dual de $C_{r}^{S}$ et $\partial^{\prime}$ le transposé de $\partial$. Alors $\partial^{\prime}$ est encore de carré nul. Le noyau $Z_{S}^{r}(M)$ (les cocycles) de $\partial^{\prime}: C_{S}^{r} \longrightarrow C_{S}^{r+1}$ contient donc l'image $B_{S}^{r}(M)$ (les cobords) de $\partial^{\prime}: C_{S}^{r-1} \longrightarrow C_{S}^{r}$ et le quotient

$$
H_{S}^{r}(M)=Z_{S}^{r}(M) / B_{S}^{r}(M)
$$

est le $r^{\text {ème }}$ espace vectoriel de cohomologie singulière réelle de $M$. Par exemple en prenant la triangulation de la Fig. 4 sur la sphère $\mathbb{S}^{2}$ 

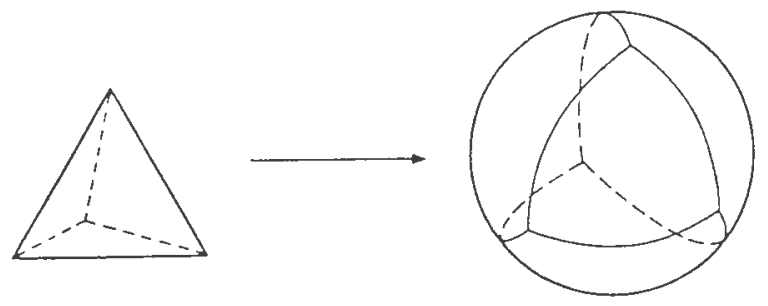

Fig. 4

on peut montrer que sa cohomologie singulière réelle est

$$
H_{S}^{r}\left(\mathbb{S}^{2}\right)= \begin{cases}\mathbb{R} & \text { si } r=0 \text { ou } 2 \\ 0 & \text { sinon }\end{cases}
$$

3. La Cohomologie de DE Rham

3.1. Les formes différentielles. En tout point $x \in M$, l'espace tangent à $M, T_{x} M$ s'identifie à un espace vectoriel réel de dimension $n=\operatorname{dim} M$. La réunion

$$
T M=\bigcup_{x \in M} T_{x} M
$$

peut être munie d'une structure de variété $C^{\infty}$ de dimension $2 n$ (une dimension $n$ qui vient de l'espace $T_{x} M$ et une autre dimension $n$ qui vient de la variété $M$ qui indexe). Un élément $p \in T M$ est de la forme $(x, u)$ où $x \in M$ et $u \in T_{x} M$. La variété $T M$ est par définition le fibré tangent à $M$. L'application $\pi: T M \longrightarrow M$ définie par $\pi(x, u)=x$ est $C^{\infty}$ et vérifie $\pi^{-1}(x)=T_{x} M$; c'est la projection canonique de $T M$ sur $M$. 
Toute application $C^{\infty}, X: M \longrightarrow T M$ vérifiant $\pi \circ X=$ $i d_{M}$ (on dit section de $T M$ ) est appelée champ de vecteurs sur $M$. Un champ de vecteurs $X$ peut etre vu comme une dérivation sur $M$ : à toute fonction $f: M \longrightarrow \mathbb{R}$ on associe sa dérivée $X$.f dans la direction de $X$.

Lorsque $M$ admet $n$ champs de vecteurs $X^{1}, \ldots, X^{n}$ tels que en tout point $x \in M$ le déterminant $\operatorname{det}\left(X_{x}^{1}, \ldots, X_{x}^{n}\right) \neq 0$, il existe un difféomorphisme $\Psi: T M \longrightarrow M \times \mathbb{R}^{n}$ tel que $\Psi_{x}: T_{x} M \longrightarrow\{x\} \times \mathbb{R}^{n}$ est linéaire et vérifie $p \circ \Psi=\pi$ où $p: M \times \mathbb{R}^{n} \longrightarrow M$ est la première projection. On dira que $M$ est isomorphe (via $\Psi$ ) au fibré trivial.

Il existe bien entendu des variétés qui ont un fibré tangent non trivial : la sphère $\mathbb{S}^{2}$ par exemple sur laquelle il n'existe même pas de champ de vecteurs partout non nul. Mais pour n'importe quelle variété $M$ chaque point admet un voisinage ouvert $U$ tel que la restriction de $T M$ à $U$ est isomorrphe à $U \times \mathbb{R}^{n}$ (via un isomorphisme $\Psi_{U}$ ). On dit que $\Psi_{U}$ est une trivialisation locale de $T M$. Cette trivialisation locale permet de ramener les objets géométriques provenant de $T M$ à des fonctions à valeurs vectorielles (qui se manipulent plus aisément).

Choisissons un ouvert $U$ de $M$ (difféomorphe à $\mathbb{R}^{n}$ ) trivialisant $T M$. Alors en tout point $x \in U$, le système de champs $\left(\frac{\partial}{\partial x_{1}}, \ldots, \frac{\partial}{\partial x_{n}}\right)$ constitue une base de l'espace $T_{x} M$. Tout champ de vecteurs $X$ sur $M$ défini sur $U$ s'écrit

$$
X=\sum_{i=1}^{n} a_{i}(x) \frac{\partial}{\partial x_{i}}
$$

où les $a_{i}$ sont des fonctions $C^{\infty}$ sur $U$.

Si $h: M \longrightarrow M$ est une application différentiable, son application tangente $h^{\prime}$ induit une application linéaire $h_{x}^{\prime}$ : $T_{x} M \longrightarrow T_{h(x)} M$ en tout point $x \in M$.

Pour tout $x \in M$ notons $\Lambda_{x}^{r}$ l'espace vectoriel (de dimension $C_{n}^{r}$ ) des formes $r$-linéaires alternées sur $T_{x} M$. L'ensemble 


$$
\Lambda^{r}=\bigcup_{x \in M} \Lambda_{x}^{r}
$$

peut être muni d'une structure de variété $C^{\infty}$ de dimension $C_{n}^{r}+n$; tout $\omega \in \Lambda^{r}$ est de la forme $\left(x, \omega_{x}\right)$ où $x$ est un point de $M$ et $\omega_{x}$ est une $r$-forme linéaire alternée sur $T_{x} M$. On a une projection $\pi^{r}: \Lambda^{r} \longrightarrow M$ définie par $\pi^{r}\left(x, \omega_{x}\right)=x$. On dira que $\Lambda^{*}=\bigoplus_{r=0}^{n} \Lambda^{r}$ est le fibré en algèbres extérieures sur $M$. Toute section $\omega: M \longrightarrow \Lambda^{r}$ (application $C^{\infty}$ vérifiant $\pi^{r} \circ \omega=i d_{M}$ ) est appelée $r$-forme différentielle sur $M: \omega_{x}$ dépend différentiablement de $x$. Une 0 -forme différentielle n'est rien d'autre qu'une fonction sur $M$. L'ensemble des $r$ formes différentielles $\Omega^{r}(M)$ sur $M$ est un module (à gauche) sur l'anneau $C^{\infty}(M)=\Omega^{0}(M)$ des fonctions réelles de classe $C^{\infty}$ sur $M$.

Tout ouvert $U$ trivialisant $T M$ trivialise le fibré $\Lambda^{r}$. Si $\left(d x_{1}, \ldots, d x_{n}\right)$ désigne la base duale de $\left(\frac{\partial}{\partial x_{1}}, \ldots, \frac{\partial}{\partial x_{n}}\right)$ alors en chaque point $x \in U,\left(d x_{i_{1}} \wedge \ldots \wedge d x_{i_{r}}\right)_{1<i_{1}<\ldots<i_{r} \leq n}$ est une base de $\Lambda_{x}^{r}$. Toute $r$-forme différentielle définie sur $U$ s'écrit alors

$$
\omega=\sum_{1 \leq i_{1}<\ldots<i_{r} \leq n} a_{i_{1} \ldots i_{r}}(x) d x_{i_{1}} \wedge \ldots \wedge d x_{i_{r}}
$$

où les $a_{i_{1} \ldots i_{r}}$ sont des fonctions $C^{\infty} \operatorname{sur} U$.

3.2. La différentielle extérieure. Rappelons que si $X$ est un champ de vecteurs sur $M$ alors la dérivée de $f \in C^{\infty}(M)$ dans la direction de $X$ est donnée par

$$
(X . f)(x)=(d f)\left(X_{x}\right)
$$

où $X_{x}$ est l'évaluation du champ $X$ au point $x \in M$.

Si $X$ et $Y$ sont deux champs de vecteurs sur $M,[X, Y]$ est leur commutateur. C'est un champ de vecteurs défini par sa dérivée sur toute fonction $f \in C^{\infty}(M)$ par 


$$
[X, Y](f)=(X . Y)(f)-(Y . X)(f)
$$

On vérifie facilement que si $X, Y$ et $Z$ sont trois champs de vecteurs sur $M$ on a l'identité (dite de Jacobi)

$$
[[X, Y], Z]+[[Y, Z], X]+[[Z, X], Y]=0
$$

Soit $\omega \in \Omega^{r}(M)$. On définit la différentielle extérieure de $\omega$ comme étant l'élément $d \omega \in \Omega^{r+1}(M)$ tel pour tout point $x \in$ $M$ et tout système de $r+1$ champs de vecteurs $X^{1}, \ldots X^{r+1}$ définis sur un voisinage de $x$ l'évaluation soit égale à

$$
\begin{gathered}
\sum_{i=1}^{r+1}(-1)^{i} X_{i} \cdot \omega\left(X_{x}^{1}, \ldots, \widehat{X}_{x}^{i}, \ldots, X_{x}^{r+1}\right)+ \\
\sum_{i<j}(-1)^{i+j} \omega_{x}\left(\left[X^{i}, X^{j}\right]_{x}, X_{x}^{1}, \ldots, \widehat{X}_{x}^{i}, \ldots, \widehat{X}_{x}^{j}, \ldots, X_{x}^{r+1}\right) .
\end{gathered}
$$

En écriture locale $\omega=\sum_{1 \leq i_{1}<\ldots<i_{r} \leq n} a_{i_{1} \ldots i_{r}} d x_{i_{1}} \wedge \ldots \wedge d x_{i_{r}}$ cette formule se réduit à

$$
d \omega=\sum_{i=1}^{n} \sum_{1 \leq i_{1}<\ldots<i_{r} \leq n} \frac{\partial a_{i_{1} \ldots i_{r}}}{\partial x_{i}} d x_{i} \wedge d x_{i_{1}} \wedge \ldots \wedge d x_{i_{r}}
$$

L'application $d$ est linéaire et vérifie $d^{2}=0$. On pose

$$
Z_{D R}^{r}(M)=\operatorname{Ker}\left(d: \Omega^{r}(M) \longrightarrow \Omega^{r+1}(M)\right)
$$

et

$$
B_{D R}^{r}(M)=\operatorname{Im}\left(d: \Omega_{D R}^{r-1}(M) \longrightarrow \Omega_{D R}^{r}(M)\right) .
$$

Le fait que $d^{2}=0$ implique que $B_{D R}^{r}(M) \subset Z_{D R}^{r}(M)$. Un élément de $Z_{D R}^{r}(M)$ est appelé $r$-forme fermée et un élément de $B_{D R}^{r}(M) r$-forme exacte. Le quotient 


$$
H_{D R}^{r}(M)=Z_{D R}^{r}(M) / B_{D R}^{r}(M)
$$

est le $r^{\text {ème }}$ espace vectoriel de cohomologie de de Rham de la variété $M$.

Nous venons de voir qu'il y a trois cohomologies (a priori différentes) définies sur une variété. Toutes les trois coincident en fait. Nous nous contenterons d'indiquer un seul isomorphisme : celui entre la cohomologie singulière et la cohomologie de de Rham.

\section{La dualité De DE RHaM}

Nous aurons besoin d'une notion de simplexe un peu plus fine que celle qu'on vient de donner au paragraphe 2 et qui calcule encore la cohomologie singulière de $M$.

On dira qu'un $r$-simplexe $\phi: \Delta_{r} \longrightarrow M$ est différentiable s'il existe un voisinage $U_{\Delta_{r}}$ de $\Delta_{r}$ dans $\mathbb{R}^{n+1}$ et une application différentiable $\widetilde{\phi}: U_{\Delta_{r}} \longrightarrow M$ qui prolonge $\phi$. L'ensemble des chaînes singulières différentiables réelles est un $\mathbb{R}$-espace vectoriel qu'on notera $C_{r}^{\infty}$.

Soient $\omega \in \Omega^{r}(M)$ et $\phi$ un $r$-simplexe singulier différentiable. On définit l'intégrale de $\omega$ sur $\phi$ par

$$
\int_{\phi} \omega=\int_{\Delta_{r}} \tilde{\phi}^{*} \omega
$$

où $\widetilde{\phi}^{*} \omega$ est la $r$-forme sur $U_{\Delta_{r}}$ image réciproque de $\omega$ par l'application $\widetilde{\phi}$. Par linéarité l'intégrale de $\omega$ s'étend à toute chaine différentiable sur $M$. La propriété clé (pour la dualité de de Rham) de l'intégrale d'une forme différentielle sur les chaînes est donnée par le

4.2. Théorème de Stokes. Soient c une $r$-chaîne différentiable sur $M$ et $\omega$ une $(r-1)$-forme défnie sur un voisinage de l'image de $c$ dans $M$. Alors

$$
\int_{\partial c} \omega=\int_{c} d \omega
$$


Nombreux sont les ouvrages où on peut trouver une démonstration de ce fameux théorème. On peut consulter par exemple [War] p. 144.

On a alors un morphisme

$$
I: \Omega^{r}(M) \longrightarrow\left(\text { dual de } C_{r}^{\infty}\right)
$$

défini par

$$
I(\omega)(c)=\int_{c} \omega
$$

Par le théorème de Stokes $I$ envoie forme fermée sur cocycle singulier (différentiable) et forme exacte sur un cobord singulier. Il induit alors un morphisme au niveau des cohomologies

$$
I^{*}: H_{D R}^{*}(M) \longrightarrow H_{S}^{*}(M, \mathbb{R})
$$

Le résultat fondamental est alors le

4.3. Théorème de de Rham. $I^{*}$ est un isomorphisme.

Sur $H_{D R}^{*}(M)$ et $H_{S}^{*}(M, \mathbb{R})$ on peut mettre des structures multiplicatives naturelles qui en font des algèbres et $I^{*}$ est en fait un isomorphisme d'algèbres.

\section{DEUXIÈME PARTIE}

Cette partie sera consacrée à la cohomologie $L^{2}$ d'une variété riemannienne complète. Nous commencerons par donner la définition pour terminer sur des exemples de calculs explicites.

Soit $\mathbb{D}=\{z \in \mathbb{C}:|z| \leq 1\}$ le disque unité dans le plan complexe et faisons agir dessus le groupe $\Gamma=\mathbb{Z} / p \mathbb{Z}$ au moyen de la rotation d'angle $\frac{2 \pi}{p}$. Alors le quotient $M=\mathbb{D} / \Gamma$ s'identifie à un cône de base le cercle $\mathbb{S}^{1}$ et de sommet 0 (cf. Fig. 5). 


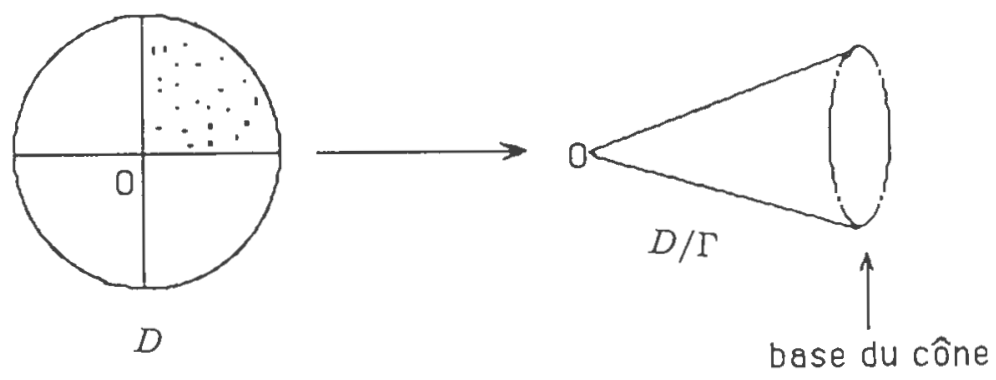

Fig. 5

On dira que 0 est une singularité conique. De manière générale une telle singularité est obtenue comme suit : soit $N$ une variété compacte; sur $N \times[0,1]$ on considère la relation d'équivalence dont les classes sont : la classe de $(x, t)$ est réduite à $\{(x, t)\}$ si $t \neq 1$ et toute la partie $N \times\{1\}$ si $t=1$. Le quotient $M$ par cette relation d'équivalence est un cône de base $N$ et de sommet la classe de $N \times\{1\}$. De manière concrète cela revient à prendre $N \times[0,1]$ et à écraser la partie $N \times\{1\}$ de façon à la réduire à un point.

D'autres espaces sont des"niveaux" de fonctions différentiables; par exemple le sous-espace dans $\mathbb{C}^{3}$ (appelé parapluie de Whitney) donné par l'équation $x^{2}-y^{2} z=0$ (voir Fig. 6)

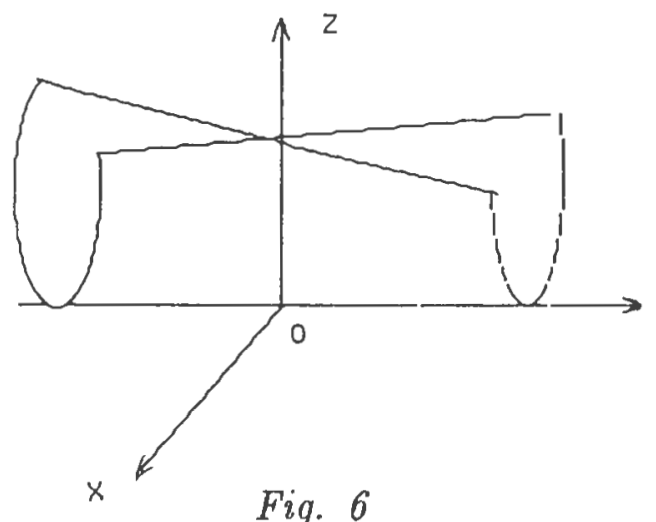

On voit aisément que les points singuliers n'admettent pas d'espace tangent; ce qui empêche l'existence de formes différen- 
tielles définies partout. Peut-on oublier les singularités et ne travailler que sur la partie régulière $M_{0}=M-\Sigma$ qui est une vraie variété $C^{\infty}$ ? Comme $M$ est supposée compacte et que le lieu singulier $\Sigma$ est fermé, $M_{0}$ est un ouvert et $\Sigma$ en est l'infini. Toutes les formes différentielles sur $M_{0}$ seraient de trop ! Il faut se contenter de celles qui ont un bon comportement près de $\Sigma$. La condition la plus naturelle est celle de croissance; la première candidate est la croissance $L^{2}$.

\section{LA COHOMOLOGIE $L^{2}$}

Soit $M$ une variété. Une métrique riemannienne sur $M$ est la donnée d'un produit scalaire $g_{x}$ sur chaque espace tangent $T_{x} M$ dépendant différentiablement de $x$. Toute variété différentiable paracompacte admet une métrique riemannienne.

5.1. L'opérateur * de Hodge. On se donne une métrique riemannienne $g$ sur une variété $M$. Si $\left(e_{1}, \ldots, e_{n}\right)$ est une base orthonormale de $T_{x}^{*} M$, alors on peut définir un produit scalaire sur chaque espace $\Lambda_{x}^{r}$ de telle sorte que la base $\left(e_{i_{1}} \wedge\right.$ $\left.\ldots \wedge e_{i_{r}}\right)_{1 \leq i_{1}<i_{2}<\ldots<i_{r} \leq n}$ soit orthonormale. On définit alors un opérateur $*: \Lambda_{x}^{r} \longrightarrow \Lambda_{x}^{n-r}$ par

$$
*\left(e_{i_{1}} \wedge \ldots \wedge e_{i_{r}}\right)=\epsilon e_{j_{1}} \wedge \ldots \wedge e_{j_{n-r}}
$$

où $\left(j_{1}, \ldots, j_{n-r}\right)$ est la suite complémentaire dans $\{1, \ldots, n\}$ et $\epsilon$ la signature de la permutation $\left\{i_{1}, \ldots, i_{r}, j_{1}, \ldots, j_{n-r}\right\}$. En opérant ainsi point par point on obtient un opérateur $C^{\infty}$ linéaire

$$
*: \Omega^{r}(M) \longrightarrow \Omega^{n-r}(M)
$$

qu'on appelle l'opérateur star de Hodge. Il vérifie : ** = $(-1)^{r(n-r)} i d$. L'image de la fonction $1 \in \Omega^{0}(M)$ par $*$ est une $n$-forme $V$ partout non nulle sur $M$ (qu'on supposera orientable). C'est la forme volume ou la mesure canonique associée à la métrique $g$. Pour toute $r$-forme $\omega$ la forme $\omega \wedge * \omega$ 
est de degré $n$ et s'écrit $f V$ où $f$ est une fonction positive sur $M$.

5.2. Définition. On dira que $\omega$ est $L^{2}$ si

$$
\int_{M} \omega \wedge * \omega<+\infty .
$$

L'ensemble $\Omega_{(2)}^{r}(M)$ des $r$-formes $L^{2}$ sur $M$ est un sousespace vectoriel de $\Omega^{r}(M)$. Il est muni d'une structure préhilbertienne donnée par le produit scalaire

$$
\langle\omega, \beta\rangle=\int_{M} \omega \wedge * \beta .
$$

Mais la différentielle extérieure d'une forme $L^{2}$ n'est pas $L^{2}$ en général (des exemples simples sur la droite réelle le montrent). Il faut se restreindre à quelque chose de plus petit. On pose (pour chaque $r=0, \ldots, n$ )

$$
\operatorname{dom} d_{r}=\left\{\omega \in \Omega_{(2)}^{r}(M): d \omega \in \Omega_{(2)}^{r+1}(M)\right\} .
$$

C'est le domaine de la différentielle $d$ opérant $\operatorname{sur} \Omega_{(2)}^{r}(M)$. Il est clair que $Z_{(2)}^{r}=\left(\operatorname{Ker}: \Omega_{(2)}^{r}(M) \longrightarrow \Omega^{r+1}(M)\right) \subset$ dom $_{r}$ et contient le sous-espace

$$
B_{(2)}^{r}=\left\{\omega \in \Omega_{(2)}^{r}(M): \omega=d \beta \text { avec } \beta \in \Omega_{(2)}^{r-1}(M)\right\} .
$$

La cohomologie $L^{2}$ de la variété $M$ est par définition

$$
H_{(2)}^{r}(M)=Z_{(2)}^{r} / B_{(2)}^{r} .
$$

Elle est associée à la métrique $g$ sur $M$ mais elle ne dépend en fait que de la classe de quasi-isométrie de $g$ (deux métriques $g$ et $g^{\prime}$ sont quasi-isométriques s'il existe une constante $k \geq 1$ telle que $\frac{1}{k} g \leq g^{\prime} \leq k g$ ). Si $M$ est compacte toute forme 
différentielle (continue par exemple) est bien sûr $L^{2}$. Dans ce cas $H_{(2)}^{*}(M)$ est exactement la cohomologie de de Rham ordinaire de $M$. L'introduction de la cohomologie $L^{2}$ ne peut donc être motivée que dans le cas non compact.

5.3. Exemple. On se contentera de la situation la plus simple, celle d'une singularité conique isolée (déjà évoquée).

Soit $N$ une variété compacte (orientable) de dimension $\mathrm{n}$ munie d'une métrique riemannienne $\widetilde{g}$. On pose

$$
C(N)=N \times] 0,1]
$$

qu'on munit de la métrique $g=\rho^{2} \widetilde{g}+d \rho^{2}$ où $\rho$ est la coordonnée sur le facteur ]0,1]. Le complété de cette variété est un espace métrique compact s’identifiant à un cône de base $N$.

Soient $M$ un espace métrique compact et $\left\{x_{1}, \ldots, x_{k}\right\}$ des points de $M$ tels que $M-\left\{x_{1}, \ldots, x_{k}\right\}$ soit une variété $C^{\infty}$ de dimension $n+1$ munie d'une métrique riemannienne. On dira que $x_{i}$ est une singularité isolée métriquement conique s'il existe une $n$-variété riemannienne compacte $\left(N_{i}, \widetilde{g}_{i}\right)$ et un voisinage $U_{i}$ de $x_{i}$ dans $M$ tels que $U_{i}-\left\{x_{i}\right\}$ soit isométrique au cône $C\left(N_{i}\right)$ qu'on vient de définir. On dira que $M$ a des singularités isolées coniques si la métrique sur $M-\left\{x_{1}, \ldots, x_{k}\right\}$ est quasi-isométrique à une métrique du type précédent. On définit alors la cohomologie $L^{2}$ de l'espace singulier $M$ par

$$
H_{(2)}^{*}(M)=H_{(2)}^{*}\left(M-\left\{x_{1}, \ldots, x_{k}\right\}\right) .
$$

Au voisinage d'une singularité (qui est un cône $C(N)$ ) on a le calcul local suivant [CGM] :

$$
H_{(2)}^{r}(C(N))= \begin{cases}H_{D R}^{r}(N) & \text { pour } r \leq \frac{n}{2} \\ 0 & \text { pour } r>\frac{n}{2}\end{cases}
$$

6. ThÉORIE DE HODGE

Comme nous l'avons signalé à l'introduction, une métrique riemannienne sur une variété compacte permet de calculer sa 
cohomologie de de Rham (qui est aussi sa cohomologie singulière) : pour tout $r=0, \ldots, n=\operatorname{dim} M$ il existe un sous-espace vectoriel $\mathcal{H}^{r}(M)$ de $\Omega^{r}(M)$ de dimension finie représentant exactement $H_{D R}^{r}(M)$. Ce résultat a été établi par W. Hodge [Hod]. Le cas non compact complet admet une version faible. L'objet de ce paragraphe est d'exposer les points les plus importants dans les deux situations.

6.1. Généralités. Soit $M$ une variété munie d'une métrique riemannienne $g$. On rappelle qu'une telle métrique permet de définir une application $C^{\infty}$-linéaire $*: \Omega^{r}(M) \longrightarrow \Omega^{n-r}(M)$ telle que $* *=(-1)^{r(n-r)} i d$. En posant : $d^{*}=(-1)^{r+1} *^{-1}$ $d *$ on obtient un opérateur de $\Omega^{r}(M)$ dans $\Omega^{r-1}(M)$ qu'on appelle la codifférentielle extérieure sur $M$. L'opérateur $\Delta=$ $d d^{*}+d^{*} d$ est d'ordre 2 et agit sur l'espace $\Omega^{r}(M)$. C'est le laplacien associé à la métrique riemannienne $g$. Une forme $\omega \in \Omega^{r}(M)$ vérifiant $\Delta \omega=0$ est dite harmonique.

Soient $U$ un ouvert (difféomorphe à $\mathbb{R}^{n}$ ) de $M$ trivialisant le fibré $\Lambda^{*}$ et $\left(x_{1}, \ldots, x_{n}\right)$ un système de coordonnées locales. Au point $x \in U$ la métrique $g$ est donnée par la matrice $\left(g_{i j}\right)_{i, j=1, \ldots, n}$ telle que $g_{i j}(x)=g_{x}\left(\frac{\partial}{\partial x_{i}}, \frac{\partial}{\partial x_{j}}\right)$.

Sur $\Omega^{0}(M)=C^{\infty}(M)$ le laplacien $\Delta$ est l'opérateur différentiel

$$
\Delta=\frac{1}{|g|^{1 / 2}} \sum_{i, j} \frac{\partial}{\partial x_{i}}\left(|g|^{1 / 2} g_{i j} \frac{\partial}{\partial x_{j}}\right)
$$

où $|g|=\operatorname{det}\left(g_{i j}\right)$. On vérifie facilement que si $M=\mathbb{R}^{n}$ muni de la métrique plate $g=\sum_{i=1}^{n} d x_{i}^{2}$ alors

$$
\Delta=\sum_{i=1}^{n} \frac{\partial^{2}}{\partial x_{i}^{2}} \text {. }
$$

Fixons $r \in\{0, \ldots, n\}$ et posons $K=C_{n}^{r}=\operatorname{rang} \Lambda^{r}$. Sur l'ouvert $U$ les $r$-formes différentielles s'interprètent comme des fonctions à valeurs dans $\mathbb{R}^{K}$ (ou $\mathbb{C}^{K}$ ). Dans ce cas le laplacien a la forme suivante 


$$
\Delta=\sum_{|I| \leq 2} a_{I}(x) D^{I}
$$

oì $I=\left(i_{1}, \ldots, i_{n}\right)$ est un multi-indice dans $\mathrm{N}^{n},|I|=i_{1}+$ $\ldots+i_{n}$ sa longueur, $D^{I}=\frac{\partial^{|I|}}{\partial x_{1}^{i 1} \ldots \partial x_{n}^{i_{n}}}$ et $a_{I}$ est une matrice carrée d'ordre $K$ à coefficients des fonctions $C^{\infty}$.

Soient $x \in U$ et $\xi=\left(\xi_{1}, \ldots, \xi_{n}\right) \in \mathbb{R}^{n}$. Alors la matrice carrée d'ordre $K$ à coefficients réels (ou complexes)

$$
\sigma(\Delta)(x, \xi)=\sum_{|I|=2} a_{I}(x) \xi_{1}^{i_{1}} \ldots \xi_{n}^{i_{n}}
$$

définit une application linéaire appelée le symbole de $\Delta$ au point $x$. Cette application est inversible pour tout point $x$ et tout vecteur $\xi$ non nul (on dit que $\Delta$ est elliptique); plus même $\sigma(\Delta)(x, \xi)$ est définie positive ( $\Delta$ est fortement elliptique). C'est l'une des propriétés majeures de l'opérateur $\Delta$ et est à la base de la théorie de Hodge.

6.2. Le cas compact. $M$ est une variété riemannienne compacte (et orientable pour simplifier).

Dans ce cas on munit $\Omega^{r}(M)$ d'une structure préhilbertienne en posant

$$
\langle\omega, \beta\rangle=\int_{M} \omega \wedge * \beta .
$$

L'opérateur $d^{*}$ devient alors l'adjoint de $d$. En effet soient $\omega \in \Omega^{r-1}(M)$ et $\beta \in \Omega^{r}(M)$. On a

$$
\begin{gathered}
d(\omega \wedge * \beta)=d \omega \wedge * \beta+(-1)^{r-1} \omega \wedge d * \beta \\
=d \omega \wedge * \beta-\omega \wedge * d^{*} \beta .
\end{gathered}
$$

En intégrant sur $M$ à gauche et à droite on obtient

$$
0=\langle d \omega, \beta\rangle-\left\langle\omega, d^{*} \beta\right\rangle
$$


On en déduit que $\Delta$ est auto-adjoint. On montre facilement que l'espace $\mathcal{H}^{r}(M)$ des $r$-formes harmoniques coincide avec l'espace Kerd $\cap \mathrm{Ker}^{*}$ i.e les formes harmoniques sont exactement les formes qui sont à la fois fermées et cofermées. On a alors pour tout $r=0, \ldots, n$ le

Théorème de Hodge. L'espace $\mathcal{H}^{r}(M)$ est de dimension finie et on a une décomposition orthogonale

$$
\Omega^{r}(M)=\mathcal{H}^{r}(M) \oplus \operatorname{Im} d \oplus \operatorname{Im} d^{*} .
$$

Comme $Z_{D R}^{r}(M)=\operatorname{Ker} d=\left(\operatorname{Im} d^{*}\right)^{\perp}$ on en déduit que $Z_{D R}^{r}(M)=\mathcal{H}^{r}(M) \oplus \operatorname{Imd}$ et donc $H_{D R}^{r}(M)=Z_{D R}^{r}(M) / \operatorname{Im} d$ s'identifie à $\mathcal{H}^{r}(M)$. Il est facile de voir aussi que

$$
\text { * }: \mathcal{H}^{r}(M) \longrightarrow \mathcal{H}^{n-r}(M)
$$

est un isomorphisme unitaire et donc $H_{D R}^{r}(M)$ et $H_{D R}^{n-r}(M)$ sont isomorphes (c'est la dualité de Poincaré).

Le théorème de Hodge est sans doute l'un des liens les plus importants entre la Topologie et l'Analyse. Sa démonstration (bien qu'actuellement elle fasse partie du folklore) est hautement non triviale et la situation où $M$ n'est pas compacte offre encore plus de difficultés.

Nous donnerons des exemples de calcul de formes harmoniques dans le cadre plus général des formes basiques d'un feuilletage riemannien.

6.3. Le cas non compact. On se restreint aux formes $L^{2}$ i.e celles qui vérifient

$$
\int_{M} \omega \wedge * \omega<+\infty
$$

Elles forment un espace préhilbertien $\Omega_{(2)}^{r}(M)$ pour le produit scalaire défini au paragraphe 5 .

Si $M$ est quelconque il n'est plus vrai que $d^{*}$ est 1'adjoint de $d$. Cela vient du fait que pour une $(n-1)$-forme $\omega$ telle 
que $\omega$ et $d \omega$ soient $L^{1}$ l'intégrale $\int_{M} d \omega$ n'est pas forcément nulle.

On dira que $M$ est complète si les géodésiques (i.e les courbes qui minimisent localement la distance) sont parmétrées de $-\infty$ à $+\infty$. Par exemple $\mathbb{R}^{n}$ muni de la métrique plate $g=$ $\sum_{i=1}^{n} d x_{i}^{2}$ est une variété riemannienne complète : en effet les géodésiques sont les droites et sont données par $x=x_{0}+t u_{0}$ où $t \in \mathbb{R}$ et $x_{0}, u_{0} \in \mathbb{R}^{n}$ fixés. Mais la variété $\mathbb{R}^{n}-\{0\}$ munie de cette même métrique n'est pas complète : les demi-droites ouvertes issues de l'origine sont des géodésiques qui ne peuvent être paramétrées (de manière affine bien sûr) que par un intervalle du type $] t_{0},+\infty[$ ou $]-\infty, t_{0}\left[\right.$ où $t_{0} \in \mathbb{R}$.

Si $(M, g)$ est complète alors $d^{*}$ est l'adjoint de $d$. Cela découle d'un résultat type Stokes dû à Gaffney [Gaf1] : pour toute $(n-1)$-forme $\omega$ sur $M$ telle que $\omega$ et $d \omega$ sont $L^{1}$ on a

$$
\int_{M} d \omega=0
$$

On en déduit que l'espace $\mathcal{H}_{(2)}^{r}(M)$ des $r$-formes harmoniques $L^{2}$ s'identifie à $\operatorname{Ker} d \cap \operatorname{Ker} d^{*}$.

Si $L_{2}^{r}(M)$ est le complété de $\Omega_{(2)}^{r}(M)$ on a une

Version faible du théorème de Hodge. ([Kod]). On a une somme orthogonale

$$
L_{2}^{r}(M)=\mathcal{H}_{(2)}^{r}(M) \oplus \overline{\operatorname{Im} d} \oplus \overline{\operatorname{Im} d^{*}}
$$

préservant $\Omega_{(2)}^{r}(M)=\Omega^{r}(M) \cap L_{2}^{r}(M)$.

Ce théorème ne donne malheureusement pas une représentation de la cohomologie $L^{2}$ par des formes harmoniques $L^{2}$ comme dans le cas compact. On a cependant une application naturelle

$$
i_{r}: \mathcal{H}_{(2)}^{r}(M) \longrightarrow H_{(2)}^{r}(M) .
$$

Lorsque $i_{r}$ est un isomorphisme on dit qu'on a un théorème fort de Hodge; c'est le cas (et c'est même équivalent) si $\overline{\operatorname{Imd}}=$ 
Im $\bar{d}$ où $\bar{d}$ est la ferméture de $d$ pour la topologie de la norme des opérateurs i.e la plus petite (pour l'inclusion des domaines) extension de $d$ à graphe fermé [CGM]. Mais on ne peut espérer avoir trop souvent cet isomorphisme; il faut se contenter de l'injectivité de $i_{r}$ qui a lieu quand la variété $M$ est complète, hypothèse dans laquelle on se mettra pour donner quelques exemples de calcul des espaces $\mathcal{H}_{(2)}^{r}(M)$. On verra aussi que l'écart entre les espaces $\mathcal{H}_{(2)}^{r}(M)$ et $H_{(2)}^{r}(M)$ peut être "assez grand".

\section{EXEMPLES DE CALCUL $L^{2}$}

On peut d'abord remarquer que *: $\mathcal{H}_{(2)}^{r}(M) \longrightarrow \mathcal{H}_{(2)}^{n-r}(M)$ est un isomorphisme unitaire. Ceci réduit de moitié le nombre d'espaces $\mathcal{H}_{(2)}^{r}(M)$ à déterminer. D'autre part comme toute forme hamonique $L^{2}$ est fermée, une fonction harmonique est nécessairement constante, donc $\mathcal{H}_{(2)}^{0}(M)=\mathcal{H}_{(2)}^{n}(M)=$ $H_{(2)}^{0}(M)=\mathbb{R}$ si le volume de $M$ est fini et les espaces $\mathcal{H}_{(2)}^{0}(M)$ et $\mathcal{H}_{(2)}^{n}(M)$ sont nuls sinon.

7.1. $M=\mathbb{R}$ avec la métrique plate $g=d x^{2}$ est une variété riemannienne complète. Comme $\operatorname{Vol}(M)=+\infty$ les espaces $\mathcal{H}_{(2)}^{0}(M)$ et $H_{(2)}^{0}(M)$ sont nuls; donc $\mathcal{H}_{(2)}^{1}(M)=0$. Mais l'espace $H_{(2)}^{1}(M)$ est loin de l'être. En effet si $f$ est une fonction à support compact $S$, la forme $\omega=f(x) d x$ est fermée et est $L^{2}$. Une primitive $\beta$ de $\omega$ est donnée par exemple par

$$
\beta(x)=\int_{m}^{x} f(x) d x
$$

où $m$ est un point de $\mathbb{R}$. La forme $\omega$ est donc exacte au sens $L^{2}$ si et seulement si la fonction $\beta$ est $L^{2}$. Mais ceci n'est pas le cas si par exemple $f$ est strictement positive à l'intérieur de $S$; en effet dans ce cas $\beta$ est positive et vaut une constante strictement positive sur un intervalle de longueur infinie. L'espace $H_{(2)}^{1}(M)$ est donc de dimension infinie. 
7.2. $M=\mathbb{S}^{1} \times \mathbb{R}$ avec $g=d \theta^{2}+d r^{2}$ (qui est complète). Alors $\operatorname{Vol}(M)=+\infty$; donc $\mathcal{H}_{(2)}^{0}(M)=\mathcal{H}_{(2)}^{2}(M)=0$. On sait que $\mathcal{H}_{(2)}^{1}(M)=\operatorname{Ker} \Delta$ où $\Delta(f d \theta+g d r)=\Delta f d \theta+\Delta g d r$ avec $\Delta=\frac{\partial^{2}}{\partial \theta^{2}}+\frac{\partial^{2}}{\partial r^{2}}$. La forme harmonique $\omega=f d \theta+g d r$ est $L^{2}$ si et seulement si $f^{2}+g^{2}$ est $L^{1}$ i.e si et seulement si $f$ et $g$ sont $L^{2}$; ce qui est encore équivalent à $f, g \in \mathcal{H}_{(2)}^{0}(M)$ i.e $f=g=0$.

Calculons $H_{(2)}^{1}(M)$. Soit $\omega=f d \theta+g d r \in \Omega_{(2)}^{1}(M)$ une forme fermée. Les fonctions $f$ et $g$ sont $L^{2}$ et vérifient $\frac{\partial f}{\partial r}=\frac{\partial g}{\partial \theta}$ (condition de ferméture de $\omega$ ). La forme $\omega$ est exacte au sens $L^{2}$ s'il existe une fonction $\beta$ qui est $L^{2}$ telle que $\frac{\partial \beta}{\partial \theta}=f$ et $\frac{\partial \beta}{\partial r}=g$. En écrivant $f(\theta, r)=\sum_{n \in \mathbb{Z}} f_{n}(r) e^{2 i \pi n \theta}$ (de même pour $g$ et $\beta$ ) ces équations deviennent

$$
\left\{\begin{aligned}
2 i \pi n \beta_{n}(r) & =f_{n}(r) \\
\beta_{n}^{\prime}(r) & =g_{n}(r)
\end{aligned}\right.
$$

La première équation exige $f_{0}(r)=0$; elle donne

$$
\beta_{n}(r)=\frac{1}{2 i \pi n} f_{n}(r) \quad \text { pour } \quad n \neq 0
$$

et $\beta_{0}$ quelconque si on suppose admise la condition $f_{0}=$ 0 . Dans ce cas l'équation (2) est automatiquement satisfaite (découle de la condition de ferméture de la forme $\omega$ ). Une forme fermée $\omega=f d \theta+g d r$ est donc exacte si et seulement $f_{0}=0$. L'espace $H_{(2)}^{1}(M)$ s'identifie donc à l'espace $\Omega_{(2)}^{0}(\mathbb{R})$ des fonctions $C^{\infty}$ de carré intégrable sur $\mathbb{R}$.

7.3. $M=\mathbb{D}$, disque unité muni de la métrique de Poincaré

$$
g=\frac{d x^{2}+d y^{2}}{\left(1-x^{2}-y^{2}\right)^{2}}
$$

Encore une fois $\operatorname{Vol}(M)=+\infty$; d'où $\mathcal{H}_{(2)}^{0}(M)=\mathcal{H}_{(2)}^{2}(M)$ qui est nul. 
Calculons $\mathcal{H}_{(2)}^{1}(M)$. Soit $\omega=a d x+b d y$ une 1-forme . Alors un calcul simple montre que $\omega \wedge * \omega=\left(a^{2}+b^{2}\right) d x \wedge d y$. Donc $\omega$ est $L^{2}$ si et seulement si la fonction $a^{2}+b^{2}$ est intégrable pour la mesure de Lebesgue sur $\mathbb{D}$. D'autre part $\omega$ est harmonique si et seulement si elle est fermée et cofermée, c'est-à-dire

$$
\frac{\partial a}{\partial y}=\frac{\partial b}{\partial x} \quad \text { et } \quad \frac{\partial a}{\partial x}=-\frac{\partial b}{\partial y}
$$

Si on pose $u=a+i b$ alors les conditions (*) sont celles de Cauchy-Riemann; elles disent que $u$ est holomorphe sur $\mathbb{D}$. L'espace $\mathcal{H}_{(2)}^{1}(M)$ est donc isométrique à l'espace des fonctions holomorphes de carré intégrable sur le disque unité. Il s'ensuit que $H_{(2)}^{1}(M)$ est de dimension infinie puisqu'il contient $\mathcal{H}_{(2)}^{1}(\mathbb{D})$.

7.4. Remarque. On peut se demander s'il existe un invariant type homologie qui pourrait être le dual de la cohomologie $L^{2}$ de manière similaire au cas lisse. Des travaux existent à ce sujet. On peut par exemple consulter la référence [CGM]. Pour des variétés singulières compactes provenant d'une action d'un groupe de Lie compact voir [Sag1].

\section{TROISIÈME PARTIE}

La théorie des feuilletages est l'étude qualitative des équations différentielles. Elle a été initiée par les travaux de $\mathrm{H}$. Poincaré, I. Bendixon et développée plus tard par C. Ehreshmann, G. Reeb, A. Haefliger... Depuis lors le sujet est devenu un large champ d'investigation en Mathématiques.

L'objet de cette partie est de donner quelques définitions et exemples pour faire sentir cette approche géométrique des systèmes différentiels sur les variétés et d'introduire un autre type d'espaces singuliers. Pour la simplicité de l'exposé toutes les variétés seront orientables et tous les objets considérés seront de classe $C^{\infty}$. 


\section{Feuilletages : DÉFINITIONS}

Un livre $M$ (épais comme un annuaire téléphonique) est un solide. Si on fait abstraction de son bord (qui est sa surface latérale) on peut le considérer comme un ouvert de $\mathbb{R}^{3}$; c'est une variété connexe de dimension 3 pour sa topologie habituelle. Mais on peut le considérer aussi comme réunion disjointe de ses feuilles qui, elles, sont des variétés de dimension 2 ; ce livre est donc aussi une variété de dimension 2 non connexe; ses composantes connexes sont précisément les feuilles. On dira que la variété $M$ (qui est de dimension 3 ) est munie d'une structure feuilletée de dimension 2 (c'est la dimension des feuilles) et de codimension 1 (c'est la dimension complémentaire des feuilles dans $M$ ). Cet exemple constitue le modèle local d'un feuilletage de codimension 1 sur une variété de dimension 3 : autour de chaque point on peut couper un petit morceau qu'on peut feuilleter à la manière qu'on vient de décrire mais de telle sorte que tous ces morceaux se recollent de façon compatible. Les définitions qu'on va donner préciseront le sens de tout cela.

Dans toute la suite $M$ désignera une variété de dimension $m+n$ (quand elle sera considérée de manière générale) réelle ou complexe suivant les cas.

8.1. Définition. Un feuilletage $\mathcal{F}$ de codimension $n$ sur $M$ est la donnée d'un recouvrement ouvert $\mathcal{U}=\left(U_{i}\right)_{i \in I}$ et d'une famille de difféomorphismes $\phi_{i}: U_{i} \longrightarrow \mathbb{R}^{m+n}$ tels que sur chaque intersection $U_{i} \cap U_{j} \neq \emptyset$ le changement de coordonnées

$$
\phi_{j} \circ \phi_{i}^{-1}(x, y)=\left(x^{\prime}, y^{\prime}\right)
$$

soit de la forme

$$
x^{\prime}=\phi_{i j}(x, y), \quad y^{\prime}=\gamma_{i j}(y)
$$

où $(x, y) \in \mathbb{R}^{m} \times \mathbb{R}^{n}=\mathbb{R}^{m+n}$.

Cela signifie que l'image par $\phi_{j} \circ \phi_{i}^{-1}$ de tout sous-espace horizontal d'équation $y=$ constante dans $\mathbb{R}^{m+n}$ est contenue 
dans un espace horizontal du même type. La variété $M$ est une réunion disjointe de sous-variétés connexes de dimension m. Chacune de ces sous-variétés est, appelée feuille de $\mathcal{F}$.

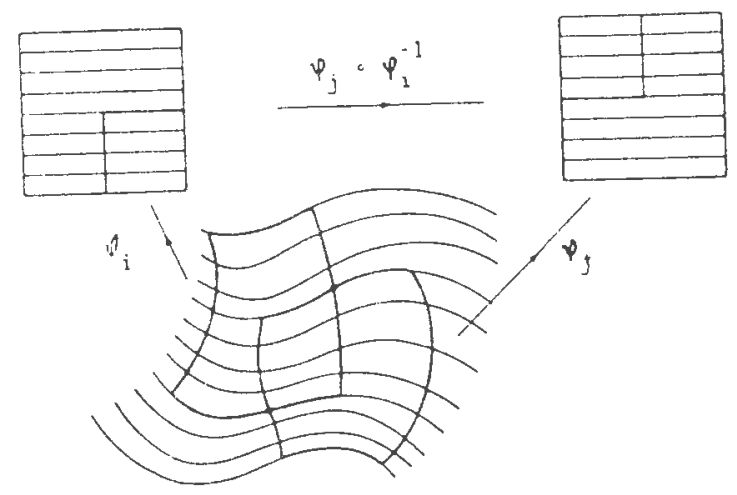

Fig. 7

Soit $\mathcal{F}$ un feuilletage de codimension $n$ sur $M$ défini par un atlas (maximal) $\left\{U_{i}, \phi_{i}\right\}_{i \in I}$ comme en 8.1. Alors l'application $f_{i}=p \circ \phi_{i}: U_{i} \longrightarrow \mathbb{R}^{n}$ où $p(x, y)=y$ est une submersion. Sur $U_{i} \cap U_{j}$ on a $f_{j}=\gamma_{i j} \circ f_{i}$.

Le recouvrement $\mathcal{U}=\left(U_{i}\right)$, les submersions $f_{i}$ et les difféomorphismes (locaux) $\gamma_{i j}$ caractérisent complètement le feuilletage $\mathcal{F}$ sur $M$.

8.2. Définition. Un feuilletage $\mathcal{F}$ sur $M$ est la donnée d'un recouvrement ouvert $\mathcal{U}=\left(U_{i}\right)_{i \in I}$, des submersions $f_{i}$ de $U_{i}$ au-dessus d'une variété transverse $T$ de dimension $n$ tels que pour $U_{i} \cap U_{j} \neq \emptyset$ il existe un difféomorphisme local $\gamma_{i j}$ de $T$ vérifiant

$$
f_{j}=\gamma_{i j} \circ f_{i}
$$

Le système $\left\{\mathcal{U}, f_{i}, \gamma_{i j}\right\}$ est appelé cocycle feuilleté définissant $\mathcal{F}$.

La démonstration de l'équivalence des définitions 8.1 et 8.2 est laissée au lecteur. 
Donnons quelques exemples pour bien visualiser comment se présente un feuilletage.

\section{Feuilletages : Exemples}

Toute variété possède un feuilletage : il suffit de prendre les points comme feuilles. Sa dimension est nulle et sa codimension est égale à celle de la variété. On peut prendre aussi le feuilletage dont la seule feuille est $M$. Il y a aussi le modèle local $\mathbb{R}^{m} \times \mathbb{R}^{n}$ d'un feuilletage de dimension $m$ et de codimension $n$.

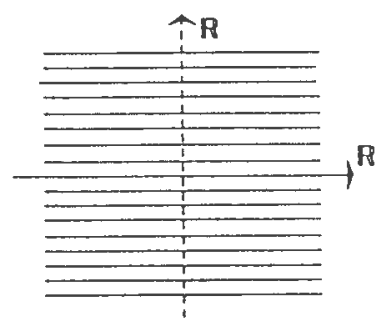

Fig. 8

Une fibration localement triviale $F \longrightarrow M \longrightarrow B$ est un feuilletage; ses feuilles sont les fibres (qui sont toutes difféomorphes à $F$ ).

Ces feuilletages constituent des cas très particuliers et n'ont pas "beaucoup d'intérêt". Nous allons en fournir d'autres un peu moins triviaux.

9.1. Feuilletages sur les surfaces. Soit $\widetilde{M}=\mathbb{R}^{2}$ et considérons l'équation différentielle

$$
d y-a d x=0 \quad \text { où } \quad a \in \mathbb{R}
$$

Elle a pour solutions $y=a x+c$ où $c \in \mathbb{R}$. En faisant varier $c$ on obtient une famille de droites parallèles définissant un 
feuilletage $\tilde{\mathcal{F}}$ de dimension 1 dans le plan.

L'action naturelle de $\mathbb{Z}^{2}$ sur $\mathbb{R}^{2}$ préserve $\widetilde{\mathcal{F}}$ (l'image de toute feuille de $\tilde{\mathcal{F}}$ par une translation entière est encore une feuille de $\tilde{\mathcal{F}}$ ). Le feuilletage $\tilde{\mathcal{F}}$ induit donc un feuilletage $\mathcal{F}$ sur le tore $\mathbb{T}^{2}=\mathbb{R}^{2} / \mathbb{Z}^{2}$. Ses feuilles sont difféomorphes au cercle $\mathbb{S}^{1}$ si $a$ est rationnel, à la droite réelle $\mathbb{R}$ et sont toutes denses si $a$ est irrationnel.

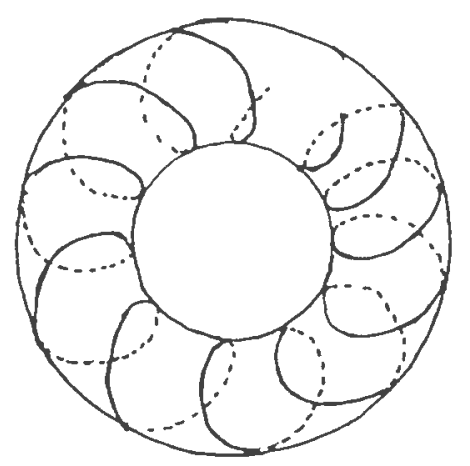

Fig. 9

Une surface fermée $M$ autre que le tore ne peut pas supporter un feuilletage de codimension 1. Le théorème de Hopf interdit par exemple l'existence de champs de vecteurs sans singularités sur $M$.

9.2. En dimension 3. i) Un exemple important de feuilletage de codimension 1 sur une variété de dimension 3, a été donné par $\mathrm{G}$. Reeb sur la sphère $\mathbb{S}^{3}$. Il constitue une réponse à une question posée par H. Hopf : est-il possible de trouver sur $\mathbb{S}^{3}$ un champ de vecteurs sans singularités $X$ tel que $X \operatorname{rot} X=0$ ? Par le théorème de Frobenius (que nous allons énoncer par la suite) ceci équivaut à l'existence d'un feuilletage de codimension 1 sur $\mathbb{S}^{3}$. On a

$$
\mathbb{S}^{3}=\left\{\left(z_{1}, z_{2}\right) \in \mathbb{C}^{2}:\left|z_{1}\right|^{2}+\left|z_{2}\right|^{2}=1\right\}
$$

Les parties 


$$
\begin{aligned}
& M_{+}=\left\{\left(z_{1}, z_{2}\right) \in \mathbb{S}^{3}:\left|z_{1}\right|^{2} \leq \frac{1}{2}\right\} \\
& M_{-}=\left\{\left(z_{1}, z_{2}\right) \in \mathbb{S}^{3}:\left|z_{2}\right|^{2} \leq \frac{1}{2}\right\}
\end{aligned}
$$

sont difféomorphes au tore solide $\overline{\mathbb{D}} \times \mathbb{S}^{1}$ où $\mathbb{D}$ est le disque unité ouvert de $\mathbb{C}$. Elles ont le tore $\mathbb{T}^{2}$ comme bord commun

$$
\partial M_{+}=\partial M_{-}=\left\{\left(z_{1}, z_{2}\right) \in \mathbb{S}^{3}:\left|z_{1}\right|^{2}=\left|z_{2}\right|^{2}=\frac{1}{2}\right\} .
$$

Réciproquement la sphère $\mathbb{S}^{3}$ peut être obtenue en recollant $M_{+}$et $M_{-}$le long de leur bord à l'aide du difféomorphisme

$$
\begin{aligned}
& h: \partial M_{+} \longrightarrow \partial M_{-} \\
& \left(z_{1}, z_{2}\right) \longrightarrow\left(z_{2}, z_{1}\right)
\end{aligned}
$$

i.e on identifie les points $\left(z_{1}, z_{2}\right)$ et $\left(z_{2}, z_{1}\right)$ dans la réunion disjointe de $M_{+}$et $M_{-}$.

Soit $f: \mathbb{D} \longrightarrow \mathbb{R}$ définie par $f(z)=e^{\frac{1}{1-|z|^{2}}}$. Elle admet la surface $S$ (de $\mathbb{R}^{3}$ ) comme graphe (voir Fig. 10)

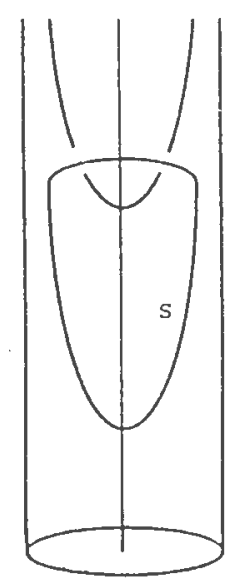

Fig. 10 
La famille des surfaces $\left(S_{t}\right)_{t \in \mathbb{R}}$ obtenues en translatant $S$ le long de l'axe vertical définit un feuilletage sur $\mathbb{D} \times \mathbb{R}$. Si on rajoute le cylindre $\mathbb{S}^{1} \times \mathbb{R}$ où $\mathbb{S}^{1}$ est vu comme le bord de $\overline{\mathbb{D}}$ on obtient un feuilletage $\widetilde{\mathcal{F}_{0}}$ sur $\overline{\mathbb{D}} \times \mathbb{R}$ invariant par la transformation

$$
\begin{gathered}
\overline{\mathbb{D}} \times \mathbb{R} \longrightarrow \overline{\mathbb{D}} \times \mathbb{R} \\
(z, t) \longrightarrow(z, t+1)
\end{gathered}
$$

et définit donc un feuilletage $\mathcal{F}_{0}$ sur le quotient (voir Fig. 11)

$$
\overline{\mathbb{D}} \times \mathbb{R} /(z, t) \sim(z, t+1) \simeq \overline{\mathbb{D}} \times \mathbb{S}^{1} .
$$

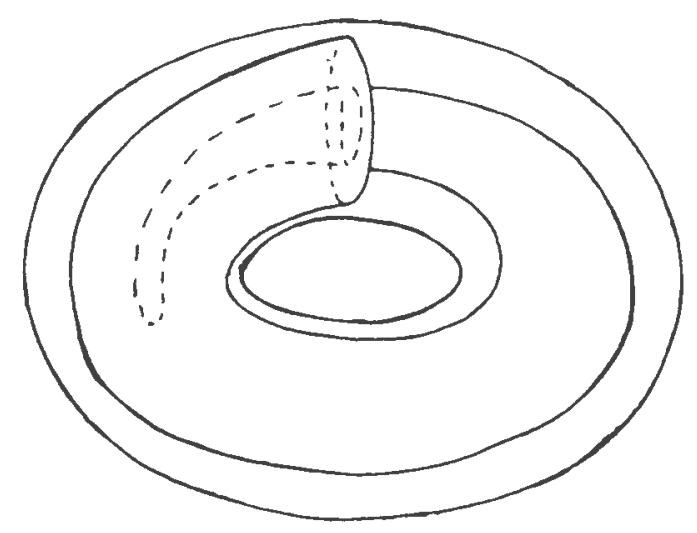

Fig. 11

Il a le bord $\mathbb{S}^{1} \times \mathbb{S}^{1}$ comme feuille compacte; les autres sont toutes difféomorphes au plan $\mathbb{R}^{2}$. Comme $M_{+}$et $M_{-}$sont difféomorphes à $\overline{\mathbb{D}} \times \mathbb{S}^{1}, \mathcal{F}_{0}$ y définit respectivement deux feuilletages $\mathcal{F}_{+}$et $\mathcal{F}_{-}$qui donnent un feuilletage sur $\mathbb{S}^{3}$ appelé le feuilletage de Reeb.

ii) Soit $A_{0} \in \mathrm{SL}(2, \mathbb{Z})$ de trace strictement supérieure à 2 . La matrice de $\mathrm{SL}(3, \mathbb{Z})$

$$
\left(\begin{array}{cc}
1 & 0 \\
0 & A_{0}
\end{array}\right)
$$


a trois valeurs propres : 1 et deux autres $\lambda$ et $\frac{1}{\lambda}$ positives irrationnelles. Soient $u=(1,0,0)$ et $v=(0,1, a)$ les vecteurs propres associés respectivement aux valeurs propres 1 et $\lambda$ et notons $\Gamma$ le sous-groupe de $\operatorname{Diff}\left(\mathbb{R}^{3}\right)$ (les difféomorphismes de $\mathbb{R}^{3}$ ) engendré par

$$
\begin{gathered}
\gamma_{1}(t, x, y)=(t, x+1, y) \\
\gamma_{2}(t, x, y)=(t, x, y+1) \\
\gamma_{3}(t, x, y)=\left(t+1, A_{0}(x, y)\right) .
\end{gathered}
$$

Ce groupe agit librement, proprement et discontinûment sur $\mathbb{R}^{3}$ et le quotient $\mathbb{R}^{3} / \Gamma$ est une variété compacte de dimension 3. Elle peut être aussi obtenue en identifiant les points $(0, x, y)$ et $\left(1, A_{0}(x, y)\right)$ dans le produit $[0,1] \times \mathbb{T}^{2}$. C'est donc un fibré plat en tores $\mathbb{T}^{2}$ au-dessus du cercle $\mathbb{S}^{1}$. Sa monodromie est donnée par la matrice $A_{0}$.

Les plans parallèles à la direction engendrée par $u$ et $v$ définissent un feuilletage $\tilde{\mathcal{F}}$ sur $\mathbb{R}^{3}$. Il est facile de voir que $\widetilde{\mathcal{F}}$ est préservé par tout $\gamma \in \Gamma$. Il induit donc un feuilletage $\mathcal{F}$ sur le quotient $\mathbb{T}_{A_{0}}^{3}=\mathbb{R}^{3} / \Gamma$. Ses feuilles sont des plans et des cylindres (qui correspondent aux points périodiques de l'action de $A_{0}$ en tant que difféomorphisme du tore $\mathbb{T}^{2}$ ).

9.3. Les suspensions des difféomorphismes. Soient $B$ et $F$ deux variétés de dimensions respectives $m$ et $n$. Supposons que le groupe fondamental $\pi_{1}(B)$ est finiment engendré et qu'on a une représentation

$$
\rho: \pi_{1}(B) \longrightarrow \operatorname{Diff}(F)
$$

où $\operatorname{Diff}(F)$ est le groupe des difféomorphismes de $F$. Notons $\widetilde{B}$ le revêtement universel de $B$ et $\widetilde{\mathcal{F}}$ le feuilletage horizontal sur $\widetilde{M}=\widetilde{B} \times F$ i.e dont les feuilles sont les parties de la 
forme $\widetilde{B} \times\{x\}$ où $x \in F$. Ce feuilletage est invariant par les transformations de $\widetilde{M}$

$$
T_{\gamma}(\tilde{b}, x)=(\gamma \cdot \tilde{b}, \rho(\gamma) \cdot x)
$$

où $\tilde{b} \in \widetilde{B}, x \in F$ et $\gamma . \tilde{b}$ désigne l'action de $\gamma \in \pi_{1}(B)$ sur $\widetilde{B}$. $\tilde{\mathcal{F}}$ induit donc un feuilletage $\mathcal{F}_{\rho}$ sur la variété quotient $M=\widetilde{M} /(\tilde{b}, x) \sim(\gamma . \tilde{b}, \rho(\gamma) \cdot x)$.

Par exemple si $B=\mathbb{S}^{1}$ et $F=[0,+\infty[$, on considère la repésentation

$$
\begin{gathered}
\rho: \pi_{1}(B)=\mathbb{Z} \longrightarrow \operatorname{Diff}([0,+\infty[) \\
1 \longrightarrow \phi
\end{gathered}
$$

où $\phi(x)=\frac{x}{2}$. Alors la variété $\widetilde{M}=\mathbb{R} \times[0,+\infty[$ est feuilletée par les droites horizontales $\mathbb{R} \times\{x\}$ où $x \in[0,+\infty[$. Le feuilletage quotient $\mathcal{F}_{\rho}$ sur $M \simeq \mathbb{S}^{1} \times[0,+\infty[$ a une feuille compacte difféomorphe au cercle et autour de laquelle s'enroulent toutes les autres feuilles (voir Fig. 12).

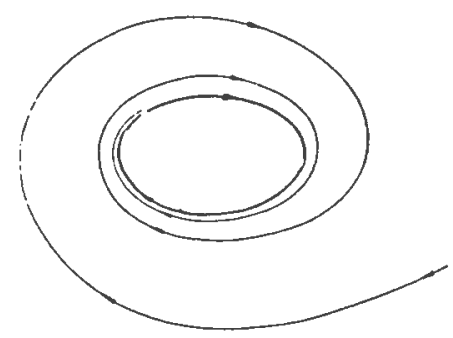

Fig. 12

10. Feullletages et Systèmes difFÉrentiels

Soit $E$ un sous-fibré de rang $m$ du fibré tangent $T M$. Ceci signifie qu'en tout point $x \in M, E_{x}$ est un sous-espace vectoriel de $T_{x} M$ de dimension $m$. Localement $T M \simeq U \times \mathbb{R}^{N}$ où 
$N=m+n$ et $U$ est un ouvert de $M$ difféomorphe à $\mathbb{R}^{N}$. Le sous-espace $E_{x}$ peut être défini comme le noyau de $n$ formes linéaires $\omega_{1}, \ldots, \omega_{n}$ linéairement indépendantes i.e

$$
E_{x}=\bigcap_{j=1}^{n} \operatorname{Ker} \omega_{j}(x)
$$

On dira que $E$ est complètement intégrable si par tout point $x \in M$ il existe une sous-variété de codimension $n, P_{x}$ admettant $E_{\mid P_{x}}$ (la restriction de $E$ à $P_{x}$ ) comme espace tangent. Les sous-variétés connexes maximales qui satisfont cette propriété définissent une partition de $M$ i.e un feuilletage de codimension $n$. On a le

10.1. Théorème de Frobenius. Soit $E$ un sous-fibré de $T M$ donné localement par le système $(S)$. Les propositions suivantes sont équivalentes

i) E est complètement intégrable,

ii) si $X$ et $Y$ sont des champs de vecteurs sur $M$ tangents $\grave{a} E$, alors le crochet $[X, Y]$ est aussi tangent $\grave{a} E$,

iii) il existe des 1-formes différentielles (définies localement) $\left(\beta_{i j}\right)_{i, j}$ telles que

$$
d \omega_{i}=\sum_{j=1}^{n} \beta_{i j} \wedge \omega_{j} \quad i=1, \ldots, n .
$$

10.2. Exemple. Soit $\omega$ une 1-forme partout non singulière sur $M$. Le sous-fibré $E$ correspondant a pour fibre au point $x \in M$ le sous-espace de codimension 1

$$
E_{x}=\operatorname{Ker} \omega(x)
$$

Le sous-fibré $E$ définit un feuilletage sur $M$ si et seulement si il existe une 1 -forme $\beta$ telle que

$$
d \omega=\beta \wedge \omega .
$$


Ce qui est équivalent ̀̀

$$
d \omega \wedge \omega=0 .
$$

En particulier si $\omega$ est fermée (i.e $d \omega=0$ ) elle définit un feuilletage $\mathcal{F}$ de codimension 1. Si $M$ est compacte toutes les feuilles sont difféomorphes. L'intégration de $\omega$ au-dessus des lacets de $M$ donne un morphisme

$$
h: \pi_{1}(M) \longrightarrow \mathbb{R}
$$

L'image $\Gamma$ de $\pi_{1}(M)$ par $h$ est un sous-groupe de $\mathbb{R}$ appelé le groupe d'holonomie de $\mathcal{F}$. L'exemple 9.1. est de ce type : $M=\mathbb{T}^{2}$ et $\omega=d y-a d x$ qui est clairement une 1-forme fermée. Le groupe fondamental de $\mathbb{T}^{2}$ est isomorphe à $\mathbb{Z}^{2}$ et il est facile de voir que $\Gamma=\{r+s a: r, s \in \mathbb{Z}\}$.

\section{Structures transverses}

Soit $\mathcal{F}$ un feuilletage de codimension $n$ sur $M$ défini par un cocycle feuilleté $\left\{U_{i}, f_{i}, \gamma_{i j}\right\}$ où $\left(U_{i}\right)_{i \in I}$ est un recouvrement ouvert de $M, f_{i}: U_{i} \longrightarrow T$ une submersion au-dessus d'une $n$-variété transverse $T$ et $\gamma_{i j}$ des difféomorphismes locaux de $T$ tels que sur $U_{i} \cap U_{j} \neq \emptyset$ on ait $f_{j}=\gamma_{i j} \circ f_{i}$ comme dans la définition 8.2 .

11.1. Définition. Une structure transverse à $\mathcal{F}$ est une structure sur $T$ invariante par les difféomorphismes locaux $\gamma_{i j}$.

Donnons quelques exemples de structures transverses. Nous nous limiterons à quelques-unes seulement.

11.2. Exemples. i) Si $T$ est un groupe de Lie et les $\gamma_{i j}$ des translations à gauche on dira que $\mathcal{F}$ est un feuilletage de Lie.

ii) On dira qu'un champ de vecteurs $Y$ sur $M$ est feuilleté si pour tout champ de vecteurs $X$ tangent à $\mathcal{F}$, le crochet $[X, Y]$ est encore tangent à $\mathcal{F}$. Le flot local associé à un tel champ préserve le feuilletage $\mathcal{F}$. Les champs tangents à $\mathcal{F}$ forment un idéal $\Gamma(\mathcal{F})$ du module $\chi(M, \mathcal{F})$ des champs feuilletés sur $M$. Le quotient $\chi(M / \mathcal{F})=\chi(M, \mathcal{F}) / \Gamma(\mathcal{F})$ est une algèbre 
de Lie appelée l'algèbre des champs basiques de $\mathcal{F}$. On dira que $\mathcal{F}$ est transversalement parallélisable (T.P en abrégé) si $\chi(M / \mathcal{F})$ est libre de rang $n$ (qui est la codimension de $\mathcal{F}$ ). Cela signifie que la variété $T$ admet un parallélisme invariant par les $\gamma_{i j}$.

iii) Supposons que $T$ est une variété riemannienne et que les $\gamma_{i j}$ sont des isométries locales. On dira alors que $\mathcal{F}$ est un riemannien. Ceci signifie que le fibré normal $\nu \mathcal{F}=T M / T \mathcal{F}$ supporte une métrique invariante le long des feuilles ou encore que la distance entre les feuilles est localement constante. Si $\left(x_{1}, \ldots, x_{m}, y_{1}, \ldots, y_{n}\right)$ sont des coordonnées locales autour d'un point $z \in M$ telles que (localement) le feuilletage soit défini par les équations $d y_{1}=\ldots=d y_{n}=0$ la métrique sur $\nu \mathcal{F}$ s'écrit

$$
g(x, y)=\sum_{i, j} g_{i j}(y) d y_{i} \otimes d y_{j}
$$

Pour une variété $M$, tout feuilletage de Lie est transversalement parallélisable et tout feuilletage transversalement parallélisable est évidemment riemannien.

La structure de chacun de ces feuilletages sur $M$ compacte est décrite comme suit.

Théorème (i) [Fed]. Supposons $\mathcal{F}$ de Lie de groupe $G$ (qui joue le rôle de $T$ ). Alors il existe un morphisme $h$ de $\pi_{1}(M)$ dans $G$ et une fibration localement triviale $D: \widetilde{M} \longrightarrow G$, où $\widetilde{M}$ est le revêtement universel de $M$ tels que

1) le feuilletage $\widetilde{\mathcal{F}}$ relevé de $\mathcal{F}$ à $\widetilde{M}$ est défini par la fibration $D$ (i.e ses feuilles sont les fibres de $D$ ),

2) pour tout $\widetilde{x} \in \widetilde{M}$ et tout $\gamma \in \pi_{1}(M)$ on a $D(\gamma \cdot \widetilde{x})=$ $h(\gamma) \cdot D \widetilde{x}$

On dira que $D$ est l'application développante de $\mathcal{F}$. L'image $\Gamma$ de $\pi_{1}(M)$ par $h$ est un sous-groupe de $G$ appelé le groupe $d$ 'holonomie de $\mathcal{F}$; son adhérence $K$ est un sous-groupe cocompact de $G$ (i.e l'espace homogène $G / K$ est compact). 
Théorème (ii) [Mol]. Supposons $\mathcal{F}$ transversalement parallélisable. Alors

1) l'adhérence de toute feuille de $\mathcal{F}$ est une sous-variété de $M$,

2) il existe un groupe de Lie $G_{0}$ tel que le feuilletage $\mathcal{F}_{0}$ induit sur chaque adhérence est de Lie de groupe $G_{0}$ à feuilles denses,

3) les adhérences des feuilles forment une fibration localement triviale $\pi: M \longrightarrow W$ au-dessus d'une variété compacte $W$; le cocycle de cette fibration est à valeurs dans le groupe $\operatorname{Diff}\left(F, \mathcal{F}_{0}\right)$ des difféomorphismes de la fibre type $F$ qui respectent le feuilletage $\mathcal{F}_{0}$.

La variété $W$ est appelée la variété basique de $\mathcal{F}$ et $\pi$ : $M \longrightarrow W$ la fibration basique de $\mathcal{F}$. Si $\mathcal{F}$ est de Lie, $W$ n'est rien d'autre que l'espace homogène $G / K$.

Théorème (iii) [Mol]. Supposons $\mathcal{F}$ riemannien et pour simplifier transversalement orientable. Notons $p: M^{\#} \longrightarrow M$ le $S O(n)$-fibré principal des repères orthonormés directs transverses $\dot{a} \mathcal{F}$. Alors $\mathcal{F}$ se relève à $M^{\#}$ en un feuilletage $\mathcal{F}^{\#}$ tel que

1) $\operatorname{dim} \mathcal{F}^{\#}=\operatorname{dim} \mathcal{F}$ et $\mathcal{F}^{\#}$ est T.P,

2) $\mathcal{F}^{\#}$ est invariant par l'action naturelle de $S O(n)$ sur $M^{\#}$.

La variété basique de $\mathcal{F}^{\#}$ sera par définition la variété basique de $\mathcal{F}$.

iv) Si $T$ est une variété analytique complexe et les $\gamma_{i j}$ des transformations biholomorphes on dira que $\mathcal{F}$ est un feuilletage transversalement holomorphe. Dans cette situation le fibré normal $\mathcal{F}$ hérite d'une structure presque complexe i.e un automorphisme

$$
J: \nu \mathcal{F} \longrightarrow \nu \mathcal{F}
$$

invariant le long des feuilles et vérifiant $J^{2}=-i d$. 
Si en plus $\mathcal{F}$ est riemannien (de métrique transverse $g$ ) on pose

$$
h\left(Y_{1}, Y_{2}\right)=\frac{1}{2}\left\{g\left(J Y_{1}, J Y_{2}\right)+g\left(Y_{1}, Y_{2}\right)\right\} .
$$

pour toutes sections $Y_{1}, Y_{2}$ de $\nu \mathcal{F}$. Clairement $h$ satisfait la condition

$$
h\left(J Y_{1}, J Y_{2}\right)=h\left(Y_{1}, Y_{2}\right)
$$

i.e $h$ est une métrique hermitienne transverse à $\mathcal{F}$. On dira que le feuilletage $\mathcal{F}$ est hermitien.

Un cas particulier de feuilletage transversalement holomorphe est donné par un feuilletage holomorphe : la variété $M$ est analytique complexe et les subrnersions $f_{i}: U_{i} \longrightarrow T$ sont holomorphes. Donnons un exemple concret, d'un tel feuilletage. Soit $M=\mathbb{C}^{2}-\{0\}$ et considérons le champ de vecteurs holomorphe $Z$ défini en tout point $\left(z_{1}, z_{2}\right) \in \mathbb{C}^{2}$ par

$$
Z\left(z_{1}, z_{2}\right)=\alpha z_{1} \frac{\partial}{\partial z_{1}}+\beta z_{2} \frac{\partial}{\partial z_{2}}
$$

où $\alpha, \beta \in \mathbb{C}^{*}$. Les variétés intégrales de $Z$ définissent un feuilletage holomorphe de codimension complexe 1 sur $M$.

Si $\frac{\alpha}{\beta} \notin \mathbb{R}^{-}$, le champ $Z$ intersecte transversalement la sphère $\mathbb{S}^{3} \subset \mathbb{C}^{2}$ et y définit un flot réel (i.e un feuilletage de dimension réelle 1 ) transversalement holomorphe. Il a deux orbites fermées $L_{1}$ et $L_{2}$ (difféomorphes au cercle) correspondant respectivement à $z_{1}=0$ et à̀ $z_{2}=0$.

\section{QUATRIÈME PARTIE}

On a vu qu'un feuilletage $\mathcal{F}$ sur $M$ est la réalisation géométrique d'un système différentiel complètement intégrable $S$ sur $M$. Chaque variété intégrale est une feuille de $\mathcal{F}$ et correspond à une condition initiale de $S$. On peut donc considérer l'espace des feuilles $Q=M / \mathcal{F}$ comme un espace de paramètres des conditions initiales de ce système différentiel. En général $Q$ 
n'est pas une variété. On peut tout de même définir dessus "certaines structures géométriques"; et on peut alors se de-mander : dans quelle mesure l'espace $Q$ ressemble-t-il d̀ une bonne variété? (du moins du point de vue cohomologique).

\section{Cohomlogie Basique}

Soient $T M, T \mathcal{F}$ et $\nu \mathcal{F}=T M / T \mathcal{F}$ respectivement le fibré tangent à $M$, le fibré tangent à $\mathcal{F}$ et son fibré normal; $\nu_{\mathcal{F}}^{*}$ sera le fibré dual de $\nu \mathcal{F}$. Les sections $\alpha$ du fibré $\Lambda^{r} \nu_{\mathcal{F}}^{*}$ vérifiant $L_{X} \alpha=0$ pour tout champ de vecteurs $X$ tangent à $\mathcal{F}$ sont les $r$-formes basiques de $\mathcal{F}$. Elles forment un module $\Omega^{r}(M / \mathcal{F})$ sur l'anneau $A(M / \mathcal{F})$ des fonctions basiques sur $M$ i.e constantes sur les feuilles de $\mathcal{F}$. La différentielle extérieure d'une forme basique est basique; la cohomologie associée au complexe différentiel $\left(\Omega^{*}(M / \mathcal{F}), d\right)$ est appelée la cohomologie basique de la variété feuilletée $(M, \mathcal{F})$ et est notée $H^{*}(M / \mathcal{F})$. C'est un invariant topologique de $\mathcal{F}$ dans la catégorie des feuilletages riemanniens complets [EN1]. On peut l'envisager comme cohomologie de de Rham de la structure transverse, voire de l'espace des feuilles de $\mathcal{F}$. Ainsi lorsque $\mathcal{F}$ provient d'une fibration, $H^{*}(M / \mathcal{F})$ s'identifie à la cohomologie de de Rham de l'espace de base de la fibration. Que garde-t-elle alors de la cohomologie de de Rham d'une variété compacte? Presque rien si le feuilletage est quelconque. On peut par exemple se demander si les espaces vectoriels $H^{p}(M / \mathcal{F})$ sont de dimension finie. Ceci est immédiat pour $H^{0}(M / \mathcal{F})$ et $H^{1}(M / \mathcal{F})$. Mais en général il n'en est rien pour $H^{p}(M / \mathcal{F})$ avec $p \geq 2$ comme le montrent les exemples de G.W. Schwarz [Sch] et récemment ceux de K.S. Sarkaria [Sar] et E. Ghys [Ghy1]. Ces derniers ont l'avantage d'être analytiques. En plus une "complexification" de l'exemple de E. Ghys fournit un exemple de feuilletage transversalement holomorphe de codimension complexe au moins 2 pour lequel la cohomologie basique est de dimension infinie. Ce qui ne saurait se produire pour deux catégories de feuilletages : les feuilletages transversalement holomorphes (que nous avons déjà 
introduits) de codimension 1 (cf. [Elk2]) et les feuilletages riemanniens comme nous allons le voir.

Nous allons commencer par quelques exemples de calcul avant d'aborder la théorie de Hodge basique pour les feuilletages riemanniens dans la section 13 .

Dans toute cette section $\mathcal{F}$ sera un feuilletage de codimension $n$ sur une variété compacte $M$.

12.1. Feuilletages de Lie. Soient $h: \pi_{1}(M) \longrightarrow G$ le morphisme et $D: \widetilde{M} \longrightarrow G$ l'application développante définissant $\mathcal{F}$. Notons $\tilde{\mathcal{F}}$ le relevé de $\mathcal{F}$ à $\widetilde{M}$. Pour tout $\gamma \in \pi_{1}(M)$ on a un diagramme commutatif

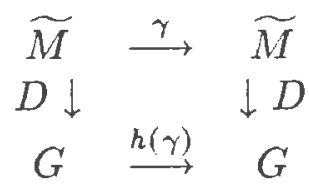

où $\gamma: \widetilde{M} \longrightarrow \widetilde{M}$ est l'automorphisme de revêtement associé à $\gamma$. Les formes basiques sur $M$ sont les formes basiques sur $\widetilde{M}$ qui sont en plus invariantes par l'action de $\pi_{1}(M)$. D'après le diagramme $(*)$, l'espace $\Omega^{*}(M / \mathcal{F})$ s'identifie de manière naturelle à l'espace des formes sur $G$ invariantes par $\Gamma=h\left(\pi_{1}(M)\right)$ et donc (par continuité) à l'espace $\Omega_{K}^{*}(G)$ des formes sur $G$ invariantes par l'adhérence $K$ de $\Gamma$ dans $G$. Les deux complexes différentiels $\left(\Omega^{*}(M / \mathcal{F}), d\right)$ et $\left(\Omega_{K}^{*}(G), d\right)$ sont donc canoniquement isomorphes; par suite leurs cohomologies respectives $H^{*}(M / \mathcal{F})$ et $H_{K^{\prime}}^{*}(G)$ sont les mêmes. En particulier si les feuilles de $\mathcal{F}$ sont denses, $K=G$ et donc $H^{*}(M / \mathcal{F})$ n'est rien d'autre que la cohomologie $H^{*}(\mathcal{G})$ de l'algèbre de Lie $\mathcal{G}$ de $G$.

Donnons un exemple concret de calcul de $H^{*}(M / \mathcal{F})$ d'une situation où $\mathcal{F}$ est à feuilles denses; le calcul dans le cas $K \neq G$ sera donné dans le cadre général des feuilletages transversalement parallélisables (T.P en abrégé).

Exemple. Soit $X=\frac{\partial}{\partial x_{0}}+a_{1} \frac{\partial}{\partial x_{1}}+\ldots+a_{n} \frac{\partial}{\partial x_{n}}$ un champ de vecteurs linéaire (i.e $a_{1}, \ldots, a_{n}$ sont des constantes réelles) sur 
le tore $\mathbb{T}^{n+1}$. Alors les orbites de $X$ définissent un feuilletage de Lie $\mathcal{F}$ de groupe $\mathbb{R}^{n}$ sur $\mathbb{T}^{n+1}$; si les nombres $1, a_{1}, \ldots, a_{n}$ sont linéairement indépendants sur $\mathbb{Q}$, les feuilles de $\mathcal{F}$ sont toutes denses. Dans ce cas on a

$$
H^{*}\left(\mathbb{T}^{n+1} / \mathcal{F}\right)=\Lambda^{*} \mathbb{R}^{n}
$$

où $\Lambda^{*} \mathbb{R}^{n}$ est l'algèbre extérieure sur $\mathbb{R}^{n}$.

On trouve le même résultat pour un feuilletage à feuilles denses défini par $m$ champs de vecteurs linéaires $X_{1}, \ldots, X_{m}$ sur le tore $\mathbb{T}^{m+n}$.

12.2. Feuilletages transversalement parallélisables. On suppose $\mathcal{F}$ T.P. Considérons sa fibration basique

$$
F \hookrightarrow M \stackrel{\pi}{\longrightarrow} W
$$

On sait (cf. 11.2. (ii)) qu'il existe un groupe de Lie 1-connexe $G_{0}$ tel que le feuilletage $\mathcal{F}_{0}$ induit sur chaque fibre $F$ de $\pi$ est de Lie de groupe $G_{0}$ et que le cocycle de la fibration $\pi$ est à valeurs dans le groupe $\operatorname{Diff}\left(F, \mathcal{F}_{0}\right)$. Notons $\mathcal{G}_{0}$ l'algèbre de Lie du groupe $G_{0}$. On sait alors qu'il existe (cf. [ESH]) une suite spectrale $\left(E_{r}\right)_{r \geq 0}$ de terme

$$
E_{r}^{p, q}=H^{p}\left(W, \mathcal{H}^{q}\right)
$$

convergeant vers $H^{*}(M / \mathcal{F})$. Ici $\mathcal{H}^{q}$ est le fibré plat de fibre $H^{q}\left(\mathcal{G}_{0}\right)$; sa monodromie est donnée par l'action de $\pi_{1}(W)$ sur $H^{q}\left(\mathcal{G}_{0}\right)$. Il découle de cette suite spectrale que l'espace vectoriel $H^{*}(M / \mathcal{F})$ est de dimension finie. L'espace $H^{n}(M / \mathcal{F})=$ $E_{2}^{s, t}$ (avec $s=\operatorname{dim} W$ et $t=\operatorname{dim} G_{0}$ ) est soit nul soit égal à $\mathbb{R}$. La situation $H^{n}(M / \mathcal{F})=0$ est effectivement possible (cf. l'exemple qui suit). Lorsque $H^{*}(M / \mathcal{F})=\mathbb{R}$ on dira que $\mathcal{F}$ est homologiquement orientable.

Exemple 1. Soit $A$ la matrice de $\mathrm{SL}(n+m, \mathbb{Z})$ 


$$
A=\left(\begin{array}{ccccc}
1 & 1 & 1 & \ldots & 1 \\
1 & 2 & 0 & \ldots & 0 \\
1 & 0 & 3 & \ldots & 0 \\
\vdots & \vdots & \vdots & \ddots & \vdots \\
1 & 0 & 0 & \ldots & d_{m+n}
\end{array}\right)
$$

où les éléments diagonaux $d_{1}, \ldots, d_{m+n}$ de $A$ sont définis par la récurrence $d_{1}=1$ et $d_{k+1}=1+d_{1} \cdot d_{2} \cdot \ldots \cdot d_{k}$ pour $k=$ $1, \ldots, m+n-1$. Alors la matrice $A$ a une valeur propre $\nu_{1}$ dans l'intervalle $(0,1)$ et exactement une valeur propre $\nu_{2}, \ldots, \nu_{m+n}$ respectivement dans chacun des intervalles $\left(d_{2}, d_{3}\right),\left(d_{3}, d_{4}\right)$, $\ldots,\left(d_{m+n}, \infty\right)$. On choisit un bloc $\left(\mu_{1}, \ldots, \mu_{m}\right)$ parmi les valeurs propres $\nu_{1}, \ldots, \nu_{m+n}$ et on notera $\lambda_{1}, \ldots, \lambda_{n}$ les autres valeurs qui restent. Soit $\left(v_{1}, \ldots, v_{m}, w_{1}, \ldots, w_{n}\right)$ une base de vecteurs propres associés respectivement à ces valeurs.

i) Pour tout $i=1, \ldots, m$, les coordonnées $v_{i}^{1}, \ldots, v_{i}^{m+n}$ de $v_{i}$ dans la base $\left(v_{1}, \ldots, v_{m}, w_{1}, \ldots, w_{n}\right)$ sont linéairement indépendantes sur $\mathbb{Q}$;

ii) tout produit $\lambda_{j_{1}} \times \ldots \times \lambda_{j_{k}}$ est différent de 1 .

La matrice $A$ définit un difféomorphisme de $\mathbb{T}^{m+n}$. Soient $X_{1}, \ldots, X_{m}, Y_{1}, \ldots, Y_{n}$ des champs de vecteurs linéaires sur $\mathbb{T}^{m+n}$ de directions respectives $v_{1}, \ldots, v_{m}, w_{1}, \ldots w_{n}$ et $\mathcal{F}_{0}$ le feuilletage sur $\mathbb{T}^{m+n}$ defini par les champs $X_{1}, \ldots, X_{m}$ qui est un feuilletage de Lie de groupe $\mathbb{R}^{n}$ et à feuilles denses. Le produit de $\mathbb{T}^{m+n}$ par $\mathbb{R}$ donne un feuilletage de codimension $n+1$ sur $\mathbb{T}^{m+n} \times \mathbb{R}$ invariant par le difféomorphisme $\phi$ de $\mathbb{T}^{m+n} \times \mathbb{R}$ qui envoie $(z, t)$ sur $(A(z), t+1)$. Il induit donc un feuilletage $\mathcal{F}$ de codimension $n+1$ sur la variété quotient $\mathbb{T}_{A}^{m+n+1}=\mathbb{T}^{m+n} \times \mathbb{R} / \phi$. Ce feuilletage est transversalement de Lie de groupe le produit semi-direct $G=\mathbb{R}^{n} \rtimes \mathbb{R}$ avec $\mathbb{R}$ agissant sur $G_{0}=\mathbb{R}^{n}$ par

$$
\begin{aligned}
& \mathbb{R} \times \mathbb{R}^{n} \longrightarrow \mathbb{R}^{n} \\
& (t, z) \longrightarrow A_{0}^{t} z
\end{aligned}
$$

où $A_{0}$ est la matrice diagonale dont les termes sont $\lambda_{1}, \ldots, \lambda_{n}$. La fibration basique est la fibration naturelle 


$$
\mathbb{T}^{m+n} \hookrightarrow \mathbb{T}_{A}^{m+n+1} \longrightarrow \mathbb{S}^{1}
$$

et la suite spectrale de cohomologie basique de $\mathcal{F}$ s'écrit

$$
E_{2}^{p, q}=H^{p}\left(\mathbb{S}^{1}, H^{q}\left(\mathcal{G}_{0}\right)\right)
$$

On a

$$
H^{q}\left(\mathcal{G}_{0}\right)=\bigoplus_{1 \leq j_{1}<\ldots<j_{q} \leq n} \mathbb{R} \eta_{j_{1}} \wedge \ldots \wedge \eta_{j_{q}}
$$

où $\left(\eta_{1}, \ldots, \eta_{n}\right)$ est la base duale de la base de $\mathbb{R}^{n}$ formée des vecteurs propres $w_{1}, \ldots, w_{n}$. L'action de $\pi_{1}\left(\mathbb{S}^{1}\right)$ sur $H^{q}\left(\mathcal{G}_{0}\right)$ est donnée par la matrice $A_{0}$ et s'écrit (pour $q \geq 1$ )

$$
A_{0}^{*}\left(\eta_{j_{1}} \wedge \ldots \wedge \eta_{j_{q}}\right)=\left(\lambda_{j_{1}} \times \ldots \times \lambda_{j_{q}}\right)\left(\eta_{j_{1}} \wedge \ldots \wedge \eta_{j_{q}}\right)
$$

sur le générateur $\eta_{j_{1}} \wedge \ldots \wedge \eta_{j_{q}}$. Comme aucun des produits $\lambda_{j_{1}} \times \ldots \times \lambda_{j_{q}}$ n'est égal à 1 on a

$$
H^{0}\left(\mathbb{S}^{1}, H^{q}\left(\mathcal{G}_{0}\right)\right)= \begin{cases}0 & \text { si } q \geq 1 \\ \mathbb{R} & \text { pour } q=0\end{cases}
$$

On rappelle que si $W$ est une variété compacte orientable et $V$ un fibré vectoriel plat au-dessus de $W$ alors la cohomologie de $W$ à valeurs dans $V$ vérifie la "dualité de Poincaré" : pour tout $p=0, \ldots, s=\operatorname{dim} W$ on a un isomorphisme $H^{p}(W, V) \cong$ $H^{s-p}\left(W, V^{*}\right)$ où $V^{*}$ désigne le fibré plat dual de $V$ (qui est trivial en tant que fibré plat si et seulement si $V$ l'est). Comme l'action de $\pi_{1}\left(\mathbb{S}^{1}\right) \operatorname{sur}\left(H^{q}\left(\mathcal{G}_{0}\right)\right)^{*}$ est donnée par la matrice $A_{0}^{-1}$ on obtient

$$
H^{1}\left(\mathbb{S}^{1}, H^{q}\left(\mathcal{G}_{0}\right)\right)=H^{0}\left(\mathbb{S}^{1},\left(H^{q}\left(\mathcal{G}_{0}\right)\right)^{*}\right)= \begin{cases}0 & \text { si } q \geq 1 \\ \mathbb{R} & \text { pour } q=0\end{cases}
$$

D'autre part comme dim $\mathbb{S}^{1}=1$, la suite spectrale dégénère au terme $E_{2}$; on a donc 


$$
H^{*}\left(\mathbb{T}_{A}^{m+n+1} / \mathcal{F}\right)= \begin{cases}\mathbb{R} & \text { si } *=0,1 \\ 0 & \text { si } * \geq 2\end{cases}
$$

qui n'est rien d'autre que la cohomologie de de Rham du cercle $\mathbb{S}^{1}$; d'ailleurs $H^{1}\left(\mathbb{T}_{A}^{m+n+1} / \mathcal{F}\right)$ est engendré par $\pi^{*}(d t)$ où $d t$ est la forme volume habituelle sur $\mathbb{S}^{\mathbf{1}}$ (base de la fibration basique de $\mathcal{F}$ ). D'autre part pour $m$ et $n$ supérieurs ou égaux à 1 on a toujours $H^{n+1}\left(\mathbb{T}_{A}^{m+n+1} / \mathcal{F}\right)=0$ i.e $\mathcal{F}$ n'est pas homologiquement orientable.

Une parenthèse. Le feuilletage $\mathcal{F}_{A}$ défini par les champs $X_{1}, \ldots, X_{m}$ et $\frac{\partial}{\partial t}$ possède de "bonnes propriétés" : il est totalement géodésique (i.e les feuilles sont des sous-variétés totalement géodésiques); $\mathcal{F}_{A}$ est $C^{\infty}$-stable i.e tout feuilletage qui lui est $C^{\infty}$-proche lui est $C^{\infty}$-conjugué (cf. [BB], [EN3] et [Ghy2]). En plus on peut démontrer facilement que sa $c o$ homologie feuilletée (i.e la cohomologie des sections du fibré $\Lambda^{*} T^{*} \mathcal{F}$ avec la différentielle extérieure le long des feuilles) vérifie la Théorie de Hodge.

Exemple 2. Notons $G A$ le groupe des transformations affines de la droite réelle qui préservent l'orientation. Ce groupe se plonge dans $\mathrm{SL}(2, \mathbb{R})$ de la façon suivante

$$
\left(\begin{array}{c}
x \longrightarrow a x+b \\
a>0
\end{array}\right) \longrightarrow \frac{1}{\sqrt{a}}\left(\begin{array}{ll}
a & b \\
0 & 1
\end{array}\right) .
$$

Il existe une variété compacte $M$ munie d'un feuilletage $\mathcal{F}$ de Lie de groupe $G=\mathrm{SL}(2, \mathbb{R})$ dont l'adhérence $K$ du groupe d'holonomie est exactement $G A$ (cf. [EN2]). La cohomologie basique de $\mathcal{F}$ est alors la cohomologie des formes sur $\mathrm{SL}(2, \mathbb{R})$ invariantes par $G A$. Le quotient $\mathrm{SL}(2, \mathbb{R}) / G A$ est difféomorphe au cercle $\mathbb{S}^{1}$. On a alors une suite spectrale

$$
E_{2}^{p, q}=H^{p}\left(\mathbb{S}^{1}, H^{q}(\mathcal{G} \mathcal{A})\right)
$$

convergeant vers $H^{*}(M / \mathcal{F})$. Comme le fibré 


$$
G A \hookrightarrow \mathrm{SL}(2, \mathbb{R}) \longrightarrow \mathbb{S}^{1}
$$

est principal et $G A$ connexe, l'action de $\pi_{1}\left(\mathbb{S}^{1}\right)$ sur $H^{q}(\mathcal{G} \mathcal{A})$ est triviale. D'où

$$
E_{2}^{p, q}=H^{p}\left(\mathbb{S}^{1}\right) \otimes H^{q}(\mathcal{G} \mathcal{A})
$$

Encore une fois comme $\operatorname{dim} \mathbb{S}^{1}=1$, cette suite spectrale converge au terme $E_{2}$. D'autre part

$$
H^{q}(\mathcal{G} \mathcal{A})= \begin{cases}\mathbb{R} & \text { pour } q=0,1 \\ 0 & \text { sinon }\end{cases}
$$

Ce qui nous donne

$$
H^{*}(M / \mathcal{F})= \begin{cases}\mathbb{R} & \text { si } *=0 \text { ou } 2 \\ \mathbb{R} \oplus \mathbb{R} & \text { si } *=1 \\ 0 & \text { sinon }\end{cases}
$$

12.3. Feuilletages riemanniens. Outre le fait d'utiliser les formes harmoniques basiques, on peut calculer la cohomologie basique d'un feuilletage riemannien par des méthodes de suites spectrales et les théorèmes de structure donnés à la section 11 . Il y a deux suites spectrales.

La première (cf. [ESH]). Supposons $\mathcal{F}$ transversalement orientable et considérons le fibré principal des repères orthonormés directs transveres à $\mathcal{F}: S O(n) \longrightarrow M^{\#} \longrightarrow M(\mathrm{Th}$. 11.2. (iii)). Alors on a une suite spectrale

$$
A_{2}^{k, l}=H^{k}(M / \mathcal{F}) \otimes H^{l}(S O(n))
$$

convergeant vers $H^{*}\left(M^{\#} / \mathcal{F}^{\#}\right)$. A l'aide de cette suite on montre que $H^{*}(M / \mathcal{F})$ est de dimension finie; elle permet aussi (par une descente) de calculer effectivement $H^{*}(M / \mathcal{F})$ quand on connait $H^{*}\left(M^{\#} / \mathcal{F}^{\#}\right)$. 
La deuxième ( $\mathrm{cf}$. [EN1]). Cette fois-ci on n'a pas besoin de remonter au fibré $S O(n) \hookrightarrow M^{\#} \longrightarrow M$ mais l'idée est la même que pour les feuilletages T.P : on utilise l'espace $X$ des adhérences des feuilles de $\mathcal{F}$. Ces adhérences sont des sous-variétés de $M$ mais à dimension variable et donc la projection canonique $\pi: M \longrightarrow X$ n'est plus une fibration localement triviale. Cependant le feuilletage $\mathcal{F}$ a un "bon comportement" au voisinage de chaque adhérence de feuille (cf. [Hae]). Ceci joint au fait que l'espace $X$ admet un bon recouvrement $\mathcal{U}=\left(U_{i}\right)_{i=1, \ldots, u}$ (i.e toute intersection $U_{i_{1}} \cap \ldots \cap U_{i_{p}}$ est contractile) donne une suite spectrale $E_{r}$ (appelée suite spectrale de cohomologie basique de $\mathcal{F})$ qui converge vers $H^{*}(M / \mathcal{F})$ et de terme

$$
E_{2}^{p, q}=H^{p}\left(\mathcal{U}, \mathcal{H}_{b}^{q}\right)
$$

où $\mathcal{H}_{b}^{q}$ est le préfaisceau qui à tout ouvert $U \subset X$ associe $H^{q}\left(\pi^{-1}(U) / \mathcal{F}\right)$ avec $\pi: M \longrightarrow X$ la projection canonique. Si $L$ est une adhérence de feuille contenue dans $\pi^{-1}(U)$ de dimension minimale, l'injection $L \hookrightarrow \pi^{-1}(U)$ induit un isomorphisme $H^{q}\left(\pi^{-1}(U) / \mathcal{F}\right) \simeq H^{q}(L / \mathcal{F})$. Comme le feuilletage induit sur $L$ est à feuilles denses sa cohomologie basique $H^{q}(L / \mathcal{F})$ est de dimension finie (car l'espace des formes différenticlles basiques lui-même est de dimension finie) et donc $H^{*}(M / \mathcal{F})$ est de dimension finie. C'est aussi cette suite spectrale qui permet de démontrer que $H^{*}(M / \mathcal{F})$ est un invariant topologique pour les feuilletages riemanniens (complets) : elle réduit le problème à celui des feuilletages riemanniens à feuilles denses.

Exemple 1. Reprenons l'exemple 11.2. iv) de flot $\mathcal{F}$ sur la sphère $\mathbb{S}^{3}$ induit par le champ holomorphe $Z=\alpha z_{1} \frac{\partial}{\partial z_{1}}+$ $\beta z_{2} \frac{\partial}{\partial z_{2}}$ avec $\frac{\alpha}{\beta} \notin \mathbb{R}^{-}$. Si $|\alpha|=|\beta|=1$, le feuilletage $\mathcal{F}$ est riemannien (il est même transversalement kählérien i.e il est transversalement modelé sur une variété kählérienne). Si $\alpha=\beta=1$, ce feuilletage n'est rien d'autre que la fibration de Hopf $\mathbb{S}^{1} \hookrightarrow \mathbb{S}^{3} \longrightarrow \mathbb{C} P^{1}$. Nous allons calculer $H^{*}\left(\mathbb{S}^{3} / \mathcal{F}\right)$. Si 
toutes les feuilles sont fermées le quotient $Q=M / \mathcal{F}$ est une variété de Sataké (ou $V$-variété) homéomorphe à $\mathbb{S}^{2}$ et donc $H^{*}\left(\mathbb{S}^{3} / \mathcal{F}\right)=H^{*}\left(\mathbb{S}^{2}\right)$. Ecartons donc ce cas. Nous utiliserons la deuxième suite spectrale; le calcul par la première se trouve par exemple dans [ESH].

Les adhérences des feuilles sont des tores $\mathbb{T}^{2}$ sauf pour les deux feuilles $L_{1}$ et $L_{2}$ correspondant respectivement à $z_{1}=0$ et $z_{2}=0$ qui sont difféomorphes au cercle $\mathbb{S}^{1}$. L'espace $X$ s'identifie à l'intervalle $[0,1]$. On prend le bon recouvrement sur $X$ formé des deux ouverts

$$
U_{1}=\left[0,2 / 3\left[\text { et } U_{2}=\right] 1 / 3,1\right]
$$

qui ont pour intersection $\left.U_{1} \cap U_{2}=\right] \frac{1}{3}, \frac{2}{3}\left[\right.$. En posant $V_{1}=$ $\pi^{-1}\left(U_{1}\right), V_{2}=\pi^{-1}\left(U_{2}\right)$ et $V_{12}=\pi^{-1}\left(U_{1} \cap U_{2}\right)$, il est facile de voir que

$$
H^{*}\left(V_{1} / \mathcal{F}\right)=H^{*}\left(V_{2} / \mathcal{F}\right)=H^{*}(\text { point })= \begin{cases}\mathbb{R} & \text { pour } *=0 \\ 0 & \text { sinon }\end{cases}
$$

et

$$
H^{*}\left(V_{12} / \mathcal{F}\right)=H^{*}\left(\mathbb{S}^{1}\right)= \begin{cases}\mathbb{R} & \text { si } *=0,1 \\ 0 & \text { sinon }\end{cases}
$$

Comme il n'y a que deux ouverts, la suite spectrale de cohomologie basique de $\mathcal{F}$ se réduit à une suite exacte longue

$$
\begin{gathered}
0 \longrightarrow H^{0}\left(\mathbb{S}^{3} / \mathcal{F}\right) \longrightarrow \mathbb{R} \oplus \mathbb{R} \longrightarrow \mathbb{R} \longrightarrow H^{1}\left(\mathbb{S}^{3} / \mathcal{F}\right) \longrightarrow \\
0 \longrightarrow \mathbb{R} \longrightarrow H^{2}\left(\mathbb{S}^{3} / \mathcal{F}\right) \longrightarrow 0
\end{gathered}
$$

Comme $H^{1}\left(\mathbb{S}^{3} / \mathcal{F}\right)$ se plonge dans $H_{D R}^{1}\left(\mathbb{S}^{3}\right)$ qui est nul on a $H^{1}\left(\mathbb{S}^{3} / \mathcal{F}\right)=0$, cette suite donne 


$$
H^{*}\left(\mathbb{S}^{3} / \mathcal{F}\right)= \begin{cases}\mathbb{R} & \text { pour } *=0,2 \\ 0 & \text { sinon }\end{cases}
$$

qui n'est rien d'autre que la cohomologie de de Rham de $\mathbb{C} P^{1}$. En particulier $H^{*}\left(\mathbb{S}^{3} / \mathcal{F}\right)$ ne dépend pas de $\alpha$ et $\beta$. L'exemple qui suit généralise cette situation à certains flots riemanniens du même type sur une sphère d'homologie $M$.

Exemple 2. Supposons que $\mathcal{F}$ soit défini par une action isométrique de $\mathbb{R}$ i.e une action localement libre de $\mathbb{R}$ sur $M$ (de dimension $n+1$ ) préservant une métrique riemannienne. C'est toujours le cas si $\mathcal{F}$ est riemannien et $M$ simplement connexe (cf. [Ghy3]). L'image de $\mathbb{R}$ par la représentation $\rho: \mathbb{R} \longrightarrow \operatorname{Isom}(M)$ (qui définit l'action) est un sous-goupe connexe abélien du groupe compact $\operatorname{Isom}(M)$; son adhérence $K$ est un sous-groupe compact connexe (un tore). La cohomologie de de Rham de $M$ est donc isomorphe à celle du complexe $\left(\Omega_{K}^{*}(M), d\right)$ où $\Omega_{K}^{*}(M)$ est l'espace des formes invariantes par l'action de $K$. Soit $X$ le champ fondamental définissant $\mathcal{F}$. On peut voir facilement que

$$
\Omega^{*}(M / \mathcal{F})=\left\{\omega \in \Omega_{K}^{*}(M): i_{X} w=0\right\} .
$$

Pour tout $r=1, \ldots, n+1$ soit $\phi: \Omega_{K}^{r}(M) \longrightarrow \Omega_{K}^{r-1}(M)$ l'application définie par $\phi(\omega)=(-1)^{r} i_{X}(\omega)$ (cf. [Sar2]). Il est facile de voir que $\phi$ est linéaire et que son image est le sous-espace vectoriel $\Omega^{r-1}(M / \mathcal{F})$; la suite

$$
0 \longrightarrow \Omega^{r}(M / \mathcal{F}) \hookrightarrow \Omega_{K}^{r}(M) \stackrel{\phi}{\longrightarrow} \Omega^{r-1}(M / \mathcal{F}) \longrightarrow 0
$$

est exacte. Elle donne une suite exacte longue de cohomologie

$$
\begin{gathered}
0 \longrightarrow H^{0}(M / \mathcal{F}) \longrightarrow H_{D R}^{0}(M) \longrightarrow H^{-1}(M / \mathcal{F})=0 \longrightarrow \\
H^{1}(M / \mathcal{F}) \longrightarrow H_{D R}^{1}(M) \longrightarrow H^{0}(M / \mathcal{F})=0 \longrightarrow \ldots
\end{gathered}
$$


$\ldots \longrightarrow H^{r}(M / \mathcal{F}) \longrightarrow H_{D R}^{r}(M) \longrightarrow H^{r-1}(M / \mathcal{F})=0 \longrightarrow \ldots$

Si $M$ est une sphère d'homologie (i.e elle a la cohomologie d'une sphère), l'entier $n$ est nécessairement pair et cette suite exacte donne

$$
H^{r}(M / \mathcal{F})= \begin{cases}\mathbb{R} & \text { si } r \text { est pair et } 0 \leq r \leq n \\ 0 & \text { sinon }\end{cases}
$$

La cohomologie basique du feuilletage $\mathcal{F}$ est donc exactement la même que celle de l'espace projectif $\mathbb{C} P^{n}$. Un exemple d'un tel flot est donné par l'intersection de la sphère $\mathbb{S}^{2 n+1} \subset \mathbb{C}^{n+1}$ avec le champ holomorphe

$$
Z=\sum_{r=1}^{n+1} \alpha_{r} z_{r} \frac{\partial}{\partial z_{r}}
$$

pour un choix convenable des complexes $\alpha_{1}, \ldots, \alpha_{n+1}$ de module 1. Pour $\alpha_{1}=\ldots=\alpha_{n+1}=1$, ce flot n'est rien d'autre que la fibration de Hopf

$$
\mathbb{S}^{1} \hookrightarrow \mathbb{S}^{2 n+1} \longrightarrow \mathbb{C} P^{n}
$$

\section{Théorie de Hodge basique}

Soit $\mathcal{F}$ un feuilletage riemannien de codimension $n$ sur une variété compacte $M$ orientable. On supposera que $\mathcal{F}$ est homologiquement orientable (et donc transversalement orientable). Cette condition est équivalente à l'existence d'une métrique riemannienne bundle-like pour laquelle les feuilles de $\mathcal{F}$ sont des sous-variétés minimales [Max] ou encore à l'existence d'une forme volume sur les feuilles $\chi$ relativement fermée i.e $d \chi\left(X_{1}, \ldots X_{\operatorname{dim} \mathcal{F}}, Y\right)=0$ (cf. [Rum]). On supposera cette hypothèse remplie. L'opérateur de Hodge habituel (cf. par exemple [Wel] ou [War]) s'étend par linéarité en un opérateur 


$$
\text { * }: \Omega^{r}(M / \mathcal{F}) \longrightarrow \Omega^{n-r}(M / \mathcal{F})
$$

pour tout $r=1, \ldots, n$. On définit d'autre part un produit scalaire sur chaque $\Omega^{r}(M / \mathcal{F})$ en posant

$$
\langle\alpha, \beta\rangle=\int_{M} \alpha \wedge * \beta \wedge \chi .
$$

Pour tout $r \in\{1, \ldots, n\}$ on pose $\delta_{r}=(-1)^{r-1} * d *$; on définit ainsi un opérateur

$$
\delta_{r}=\Omega^{r}(M / \mathcal{F}) \longrightarrow \Omega^{r-1}(M / \mathcal{F}) .
$$

Pour tous $\alpha \in \Omega^{r-1}(M / \mathcal{F})$ et $\beta \in \Omega^{n-r}(M / \mathcal{F})$ on a

$$
\begin{aligned}
d(\alpha \wedge * \beta \wedge \chi) & =d \alpha \wedge * \beta \wedge \chi+(-1)^{r-1} \alpha \wedge d(* \beta) \wedge \chi \\
& =d \alpha \wedge \beta \wedge \chi+(-1)^{r-1+r-2} \alpha \wedge *\left(\delta_{r} \beta\right) \wedge \chi \\
& =d \alpha \wedge \beta \wedge \chi-\alpha \wedge *\left(\delta_{r} \beta\right) \wedge \chi
\end{aligned}
$$

Le terme $\alpha \wedge * \beta \wedge d \chi$ est nul car $\chi$ est relativement fermée (par hypothèse). En intégrant les membres de gauche et de droite dans l'égalité qui précède on obtient

$$
0=\langle d \alpha, \beta\rangle-\left\langle\alpha, \delta_{r} \beta\right\rangle
$$

i.e $\delta_{r}$ est l'adjoint de $d: \Omega^{r-1}(M / \mathcal{F}) \longrightarrow \Omega^{r}(M / \mathcal{F})$ pour le produit scalaire qu'on vient de définir.

Pour tout $r=1, \ldots, n$ on pose

$$
\mathcal{H}^{r}(M / \mathcal{F})=\operatorname{Kerd} \cap \operatorname{Ker} \delta_{r} .
$$

On alors le théorème suivant dont on peut trouver la démonstration compète dans [Rei3], [Elk1] ou [EH]. 
13.1. Théorème. Soit $\mathcal{F}$ un feuilletage riemannien homologiquement orientable de codimension $n$ sur une variété compacte orientable $M$. Alors

i) $\operatorname{dim} \mathcal{H}^{r}(M / \mathcal{F})<+\infty$;

ii) $\Omega^{r}(M / \mathcal{F})=\mathcal{H}^{r}(M / \mathcal{F}) \oplus \operatorname{Im} d \oplus \operatorname{Im} \delta_{r+1}$.

De ce théorème on peut tirer comme conséquence importante : la dualité de Poincaré.

13.2. Théorème. L'accouplement

$$
([\alpha],[\beta]): H^{r}(M / \mathcal{F}) \otimes H^{n-r}(M / \mathcal{F}) \stackrel{\Psi}{\longrightarrow} \int_{M} \alpha \wedge \beta \wedge \chi \in \mathbb{R}
$$

est une forme bilinéaire non dégénérée et on a alors un isomorphisme canonique entre $H^{r}(M / \mathcal{F})$ et $H^{n-r}(M / \mathcal{F})$.

Ces deux théorèmes montrent que la cohomologie basique d'un feuilletage riemannien homologiquement orientable sur une variété compacte orientable se comporte comme la cohomologie de de Rham d'une "bonne variété" compacte orientable.

Le théorème 13.2 a été aussi établi par V. Sergiescu par des méthodes homologiques dans [Ser1].

En réalité la condition $H^{n}(M / \mathcal{F})=\mathbb{R}$ n'est pas nécessaire pour avoir le théorème 13.1. Nous renvoyons le lecteur aux références $[\mathrm{EH}]$ ou [Elk1] pour les démonstrations. Nous allons toutefois donner un exemple de calcul.

13.3. Exemple. Nous reprenons la matrice $A$ de l'exemple 1 du 12.2 mais en prenant (pour simplifier) $m=n=1$. La matrice $A$ a donc deux valeurs propres $\mu$ et $\lambda=\frac{1}{\mu}$ auxquelles sont associés deux vecteurs propres $v=(1, a)$ et $w=(1, b)$. La variété $\mathbb{T}_{A}^{3}$ est le quotient de $\mathbb{T}^{2} \times \mathbb{R}$ par la relation d'équivalence qui identifie $(x, y, t) \dot{\mathrm{a}}(A(x, y), t+1)$ où $(x, y, t)$ sont les coordonnées sur $\mathbb{T}^{2} \times \mathbb{R}$. Le feuilletage $\mathcal{F}$ est le flot défini par le champ de vecteurs

$$
X=\mu^{t}\left(\frac{\partial}{\partial x}+a \frac{\partial}{\partial y}\right) .
$$


Le paralléllisme transverse est donné par les champs de vecteurs

$$
Y=\lambda^{t}\left(\frac{\partial}{\partial x}+b \frac{\partial}{\partial y}\right) \text { et } Z=\frac{\partial}{\partial t} .
$$

On a les relations de crochet

$$
[X, Y]=0,[X, Z]=(-\log \mu) X \text {, et }[Y, Z]=(-\log \lambda) Y .
$$

La base duale du parallélisme $(Y, Z)$ est donnée par les formes différentielles

$$
\left\{\begin{array}{l}
\theta=\lambda^{-t}\left(\frac{a}{a-b} d x-\frac{1}{a-b} d y\right) \\
\eta=d t
\end{array}\right.
$$

dont les différentielles extérieures sont

$$
d \theta=(\log \mu) \theta \wedge \eta \text { et } d \eta=0 .
$$

Décrivons les formes basiques. Les fonctions basiques sont les fonctions qui ne dépendent que de $t$; les 1 -formes basiques sont du type

$$
\alpha=f \theta+g \eta
$$

avec $f$ et $g$ des fonctions basiques. Enfin les 2 -formes basiques s'écrivent

$$
\beta=h \theta \wedge \eta
$$

avec $h$ fonction basique. On choisit comme métrique riemannienne transverse celle qui rend le parallélisme $(Y, Z)$ orthonormé. On définit alors l'opérateur * de Hodge de la façon suivante

$$
\left\{\begin{array}{l}
*(\mathbf{1})=v \\
*(\theta)=\eta \\
*(\eta)=-\theta \\
*(v)=\mathbf{1}
\end{array}\right.
$$


où $v=\theta \wedge \eta$ et on étend $*$ linéairement par rapport aux fonctions basiques. On définit d'autre part le "morphisme d'intégration"

$$
I: \Omega^{2}\left(\mathbb{T}_{A}^{3} / \mathcal{F}\right) \longrightarrow \Omega^{1}\left(\mathbb{S}^{1}\right)
$$

$\operatorname{par} I(h(t) \theta \wedge \eta)=h(t) \eta$ qui nous permet de définir le produit scalaire sur chaque espace $\Omega^{r}\left(\mathbb{T}_{A}^{3} / \mathcal{F}\right)$ par

$$
\langle\alpha, \beta\rangle=\int_{\mathbb{S}^{1}} I(\alpha \wedge \beta) .
$$

L'adjoint $\delta: \Omega^{r}\left(\mathbb{T}_{A}^{3} / \mathcal{F}\right) \longrightarrow \Omega^{r-1}\left(\mathbb{T}_{A}^{3} / \mathcal{F}\right)$ de $d$ est défini par

$$
\begin{cases}\delta(f) & =0 \\ \delta(f \theta) & =0 \\ \delta(g \eta) & =-\frac{\partial g}{\partial t}(t) \\ \delta(h \theta \wedge \eta) & =\left(h(t) \log \mu-\frac{\partial h}{\partial t}\right) \theta\end{cases}
$$

Comme une forme basique $\omega$ est harmonique si et seulement si elle vérifie $d \omega=0$ et $\delta \omega=0$ l'expression de $\delta$ nous donne l'espace $\mathcal{H}^{*}\left(\mathbb{T}_{A}^{3} / \mathcal{F}\right)$ des formes harmoniques basiques sur $\mathbb{T}_{A}^{3}$

$$
\mathcal{H}^{*}\left(\mathbb{T}_{A}^{3} / \mathcal{F}\right)= \begin{cases}\mathbb{R} \cdot 1 & \text { si } *=0 \\ \mathbb{R} \cdot \eta & \text { si } *=1 \\ 0 & \text { si } * \geq 2\end{cases}
$$

On constante que contrairement au cas classique et au cas où $\mathcal{F}$ est homologiquement orientable, la forme volume $\theta \wedge \eta$ n'est pas harmonique même si le feuilletage est transversalement orientable. 


\section{Références}

Voici quelques références où on peut trouver les démonstrations des résultats énoncés. Certaines n'ont pas été citées dans ce texte mais contiennent beaucoup de matériel en rapport avec le sujet.

[AV]. Andreotti, A. and Vesentini, E., Carleman estimates for the LaplaceBeltrami equations, Pub. Math. I.H.E.S. (1965), 81-130.

[Ati]. Atiyah, M., Elliptic operators, discrete groups and von Neumann algebras, Asterisque 32-33 (1976), 43-72.

[APS]. Atiyah, M., Patodi, V. and Singer, I., Spectral assymetry and Riemannian geometry, I, II and III, Math. Pro. Cam. Phil. Soc., 77 (1975), 43-69, 78 (1976), 405-432 and (79 (1976)), 315-330.

[Bai]. Bailly, W., The decomposition theorem for $V$-manifolds, Amer. J. of Math., 78 (1956), 862-888.

[BB]. Belliart, M. et Birembaux, O., Actions localement libres de groupes résolubles, Prépublication Université de Valenciennes (1993).

[Cam]. Camacho, C. and Neto, A., Geometric Theory of Foliations, Birkhäuser.

[Che]. Cheeger, J., On the Hodge theory of Riemannian Pseudomanifolds, Proc. of Symp. in Pure Math., 36 (1980), 91-146.

[CGM]. Cheeger, J., Goresky, M. and Macpherson, R., $L^{2}$-Cohomology and Intersection homology, Ann. of Math. Studies, 102 (1982), 303-340.

[CM]. Connes, A. and Moscovici, H., $L^{2}$-index theorem for homogeneous spaces of Lie groups, Ann. of Math., 115, (1982), 291-330.

[Deb]. DeBaun, D., $L^{2}$-cohomology of non compact surfaces, Trans. of AMS (1984), 543-565.

[Dod1]. Dodziuk, J., Vanishing theorems for $L^{2}$-harmonic forms, Volume in memory of V.K. Patodi, Tata Institute of Fundamental Research (Bombay) (1983).

[Dod2]. Dodziuk, J., de Rham-Hodge theorry for $L^{2}$-cohomology of infinite coverings, Topology, 16 (1977), 157-165.

[Dod3]. Dodziuk, J., Sobolev spaces of differential forms and de Rham-Hodge isomorphism, J. of Diff. Geo., 16 (1981), 63-73.

[Dod4]. Dodziuk, J., $L^{2}$ - harmonic forms on rotationally symetric Riemannian manifolds, Proc. Amer. Math. Soc., 77 (1979), 395-400.

[DF]. Donneley, H. and Fefferman, C., $L^{2}$-Cohomology and Index Theorem for the Bergman metric, Ann. of Math., 118 (1983), 593-618.

[Elk1]. El Kacimi Alaoui, A., Opérateurs transversalement elliptiques sur un feuilletage riemannien et applications, Composito Mathematica, 73 (1990), 57-106.

[Elk2]. El Kacimi Alaoui, A., Dualité pour les feuilletages transversalement holomorphes, Manuscripta Math. 58 (1987), 417-443. 
[EH]. Fl Kacimi Alaoui, A et Hector, : $x$, Lcompostion de Hodge basqque

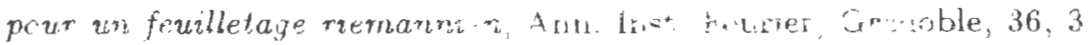
(1986). 207-227.

[ESH]. F! Kacimi Alaoui, A., Sergiescu, V. et Hector, C; I a chomonogie

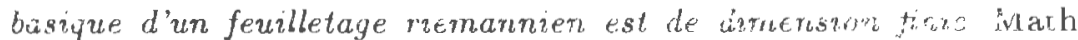
Zei., 188 (1985), 593-599.

[FN1] El Kacimi Alaoui, A. and Nicolau, M., On the topolngacai inuariance of the basic cohomology, Math. Ann., 295 (1993), 6:7 634

[EN2]. El Kacımi Alaoui, A, and Nicolau, M., Structures géométriques invariantes et feunlletages de Lie, Indagationes Mathematicae, New series, Vol 1, No. $3(1990)$, 323-334.

[EN3]. El Kacimi Alaoui, A. and Nicolau, M., A class of $C^{\infty}$-stable foliations, Ergod. Th. \& Dynam. Sys. 13 (1993), 1-8.

[Fed]. Fedida, E., Sur les feuilletages de Lie, CRAS de Paris, 272 (1971), 999-1002.

[Gaf1]. Gaffney, M., A special Stokes' Theorem for Complete Riemanrian manifolda, Ann. of Math., Vol. 60 (1954), 140-145.

[Ghy1]. Ghys, E, Un feuilletage analytique dont la cohomologie basique est de dimension infinie, Pub. IRMA - Lille, Vol. VII, Fasc. I (1985).

[Ghy2]. Ghys, E., Actıons localement libres du groupe affine, Inventiones Math., 82 (1985), 479-526.

[Ghy3]. Ghys, E., Feuilletages riemanniens sur les variétés simplement connexes, Ann. Inst. Fourier, 34, 4 (1984), 203-223.

[God]. Godbillon, C., Feuilletages. Etudes géométriques, Birkhäuser (1991).

[Gol]. Goldberg, S., Curvature and cohomology, Collection Dover.

[Hae]. Haefliger, A., Leaf closures in Riemannian foliations, A fête on Topology. Papers dedicated to Itiro Tamura; Academic Press (1988).

[HH]. Hector, G. and Hirsch, U., Introduction to the Geometry of Foliatrons, Parts A and B. Aspects of Mathematics, Vieweg.

[Hod]. Hodge, W. D., The Theory and Applications of Harmonic integrals, Camridge University Press, New York (1952).

[Kaw]. Kawasaki, T., The index of elliptic operators over V-manifolds, Nagoya Math J., Vol. 84 (1981), 135-157.

[Kit1]. Kitahara, H., Nonexistence of nontrivial $\square^{\prime \prime}$-harmonic 1-forms on a complete folzated Riemannian manifold, Trans. of AMS, 262, No. 2 (1980), 429-435.

[Kit2]. Kitahara, H., Remarks on square integrable basic cohomology spaces on a foliated Riemannian foliation, Kodai Math. J., 2 (1979), 187-193.

[Kod]. Kodaira, K., Harmonic fields in Riemannian manifolds, Annals of Math., 50 (1949), 587-665.

[Mac]. MacPherson, R., Global questions in the topology of singular spaces, ICM, Warsaw (1983). 
[Max]. Maxa. J., Duality and minimality in Riemannian foliations, Comment. Math. Helvetici, 67 (1992), 17-27.

[Mol]. Molino, P., Riemannian Foliations, Progress in Math. Vol. 73, Birkhäuser.

[ME]. Morrey, C. and Eells, J., A variational method in the theory of harmonic integrals, I, Ann. of Math., Vol. 63 (1956), 91-128.

[Nag1]. Nagase, M., $L^{2}$-Cohomology and Intersection cohomology of stratified spaces, Duke Math. J., 50 (1983), 329-368.

[Nag2]. Nagase, M., Sheaf theoretic $L^{2}$-cohomology, Advanced Stu. Pure Math. No. 8; North Holland (1987), 273-279.

[Rha]. de Rham, G., Variétés différentiables, Actualités scientifiques et Industrielles.

[Ren1]. Reinhart, B., Foliated manifolds with bundle-like metrics, Ann. of Math. , 69 (1959), 119-132.

[Ren2]. Reinhart, B., Differential Geometry of Foliations, Ergeb. der Mathematik (1983).

[Ren3]. Reinhart, B., Harmonic integrals on foliated manifolds, Amer. J. of Math., 81 (1959), 529-586.

[Rum]. Rummler, H., Quelques notions simples en géométrie riemannienne et leurs applications aux feuilletages compacts, Comment. Math. Helv., 54 (1979), 224-239.

[Sag1]. Saralegi, M., Un théorème de dualité entre l'homologie d'intersection et la cohomologie $L^{2}$, Thèse Université de Lille I (1988).

[Sag2]. Saralegi, M., The Euler class for flows of isometries, Research Notes in Math. 131. Edited by L. Cordero (1985).

[Sar]. Sarkaria, K.S., Non degenerescence of some spectral sequences, Ann. Inst. Fourier de Grenoble, 34, 1 (1984), 39-46.

[Sch]. Schwarz, G.S., On the de Rham cohomology of the leaf space of a foliation, Topology 13 (1974), 185-187.

[Ser1]. Sergiescu, V., Cohomologie basique et dualité des feuilletages riemanniens, Ann. Inst. Fourier, Grenoble 35, 3 (1985), 137-158.

[War]. Warner, W., Foundations of Differentiable Manifolds and Lie Groups, GTM 94.

[Wel]. Wells, R.O., Differential Analysis on Complex Manifolds, GTM 65 (1979).

[Zuc]. Zucker, S., Hodge theory with degenerating coefficients : $L^{2}$-Cohomology in the Poincaré Metric, Ann. of Math., 109 (1979), 415-476.

Recibido : 5 Octubre 1993

Aziz El Kacimi Alaoui

Université de Valenciennes

59326 Valenciennes Cedex, France 